\title{
Fast-timing study of ${ }^{81} \mathrm{Ga}$ from the $\beta$ decay of ${ }^{81} \mathrm{Zn}$
}

\author{
V. Paziy, ${ }^{1}$ L. M. Fraile $\odot,{ }^{1, *}$ H. Mach,${ }^{1,2, \dagger}$ B. Olaizola,,${ }^{1,}$ G. S. Simpson, ${ }^{3}$ A. Aprahamian, ${ }^{4}$ C. Bernards,${ }^{5,6}$ J. A. Briz, \\ B. Bucher, ${ }^{8}$ C. J. Chiara, $,{ }^{9} 10, \S$ Z. Dlouhý, ${ }^{11, \|}$ I. Gheorghe, ${ }^{12}$ D. Ghiţă ${ }^{12}$ P. Hoff,${ }^{13}$ J. Jolie,${ }^{5}$ U. Köster,${ }^{14}$ W. Kurcewicz, ${ }^{15}$ \\ R. Lică, ${ }^{12}$ N. Mărginean, ${ }^{12}$ R. Mărginean, ${ }^{12}$ J.-M. Régis, ${ }^{5}$ M. Rudigier,,${ }^{5, \mathbb{I}}$ T. Sava, ${ }^{12}$ \\ M. Stănoiu, ${ }^{12}$ L. Stroe, ${ }^{12}$ and W. B. Walters ${ }^{9}$ \\ ${ }^{1}$ Grupo de Física Nuclear \& IPARCOS, Facultad de Ciencias Físicas, Universidad Complutense-CEI Moncloa, E-28040 Madrid, Spain \\ ${ }^{2}$ National Centre for Nuclear Research, BP1, ul. Hoża 69, 00-681, Warsaw, Poland \\ ${ }^{3}$ LPSC, Université Joseph Fourier Grenoble 1, CNRS/IN2P3, Institut National Polytechnique de Grenoble, F-38026 Grenoble Cedex, France \\ ${ }^{4}$ Department of Physics, University of Notre Dame, Notre Dame, Indiana 46556, USA \\ ${ }^{5}$ Institut für Kernphysik, Universität zu Köln, D-50937 Köln, Germany \\ ${ }^{6}$ Wright Nuclear Structure Laboratory, Yale University, New Haven, Connecticut 06520, USA \\ ${ }^{7}$ Instituto de Estructura de la Materia, CSIC, 28006 Madrid, Spain \\ ${ }^{8}$ Idaho National Laboratory, Idaho Falls, Idaho 83415, USA \\ ${ }^{9}$ Department of Chemistry and Biochemistry, University of Maryland, College Park, Maryland 20742, USA \\ ${ }^{10}$ Physics Division, Argonne National Laboratory, Argonne, Illinois 60439, USA \\ ${ }^{11}$ Nuclear Physics Institute of the AS CR, Z-25068, Řež, Czech Republic \\ 12 “Horia Hulubei” National Institute for Physics and Nuclear Engineering, R-77125 Bucharest-Magurele, Romania \\ ${ }^{13}$ Department of Chemistry, University of Oslo, P. O. Box 1033 Blindern, N-0315 Oslo, Norway \\ ${ }^{14}$ Institut Laue-Langevin, 38042 Grenoble Cedex 9, France \\ ${ }^{15}$ Faculty of Physics, University of Warsaw, PL 02-093 Warsaw, Poland
}

(Received 11 March 2020; revised 4 June 2020; accepted 24 June 2020; published 30 July 2020)

\begin{abstract}
The $\beta^{-}$decay of ${ }^{81} \mathrm{Zn}$ to the neutron magic $N=50$ nucleus ${ }^{81} \mathrm{Ga}$, with only three valence protons with respect to ${ }^{78} \mathrm{Ni}$, was investigated. The study was performed at the ISOLDE facility at CERN by means of $\gamma$ spectroscopy. The ${ }^{81} \mathrm{Zn}$ half-life was determined to be $T_{1 / 2}=290(4) \mathrm{ms}$ while the $\beta$-delayed neutron emission probability was measured as $P_{n}=23(4) \%$. The analysis of the $\beta$-gated $\gamma$-ray singles and $\gamma-\gamma$ coincidences from the decay of ${ }^{81} \mathrm{Zn}$ provides 47 new levels and 70 new transitions in ${ }^{81} \mathrm{Ga}$. The $\beta^{-} n$ decay of ${ }^{81} \mathrm{Zn}$ was observed and a new decay scheme into the odd-odd ${ }^{80} \mathrm{Ga}$ nucleus was established. The half-lives of the first and second excited states of ${ }^{81} \mathrm{Ga}$ were measured via the fast-timing method using $\mathrm{LaBr}_{3}(\mathrm{Ce})$ detectors. The level scheme and transition rates are compared to large-scale shell-model calculations. The low-lying structure of ${ }^{81} \mathrm{Ga}$ is interpreted in terms of the coupling of the three valence protons outside the doubly magic ${ }^{78} \mathrm{Ni}$ core.
\end{abstract}

DOI: 10.1103/PhysRevC.102.014329

\section{INTRODUCTION}

Modifications to the standard ordering of the singleparticle energies have been observed in exotic nuclei with a large disparity in proton and neutron numbers. They give rise to the disappearance of the conventional magic numbers and the appearance of new shell gaps. The understanding of the underlying physics driving such modifications is one of the main subjects of modern nuclear-structure studies. It is recognized that monopole shifts are responsible for the

\footnotetext{
*1mfraile@ucm.es

${ }^{\dagger}$ Deceased.

${ }^{\ddagger}$ Present address: TRIUMF, 4004 Wesbrook Mall, Vancouver, British Columbia V6T 2A3, Canada.

${ }^{\S}$ Present address: U.S. Army Research Laboratory, Adelphi, Maryland 20783, USA.

"Deceased.

"Present address: Institut für Kernphysik, Technische Universität Darmstadt, D-64289, Darmstadt, Germany.
}

evolution of shell structure far off stability, but the effect of the different components of the monopole interaction is still the subject of investigation [1], since it is not simple to disentangle them from the experimental information. This is mainly due to the fact that effective single-particle energies (ESPEs) cannot be directly measured and that single-particle and collective effects arising from residual interactions are intertwined. The central term of the monopole interaction seems to be responsible for the evolution of ESPEs, while the tensor term plays a leading role in the splitting of spin-orbit partners.

The regions in the immediate vicinity of exotic doubly magic nuclei are key for mapping the single-particle degrees of freedom around closed cores. The evolution of the protonneutron interaction arising from the tensor force and the role of neutron excitations across neutron shell gaps can be studied in these nuclei. Relevant ingredients to theoretical models can also be obtained. Two unexplored areas in the table of nuclides still remain: around the doubly magic ${ }^{78} \mathrm{Ni}$ and in the vicinity of ${ }^{100} \mathrm{Sn}$. 
Although ${ }^{78} \mathrm{Ni}$, with 28 protons and 50 neutrons $(Z=28$, $N=50$ ), is located 14 neutrons off the stability line, it is expected to be a doubly magic nucleus due to the robust shell gaps arising from the spin-orbit splitting both for protons $\left(\pi f_{7 / 2}-\pi f_{5 / 2}\right)$ and neutrons $\left(\nu g_{9 / 2}-\nu g_{7 / 2}\right)$. The first evidence for the existence of ${ }^{78} \mathrm{Ni}$ came from [2]. Afterward its half-life was reported [3] to be $T_{1 / 2}=100_{-60}^{+100} \mathrm{~ms}$ and more recently $T_{1 / 2}=122 \pm 5 \mathrm{~ms}$ [4]. The latter value does point toward the magic character of ${ }^{78} \mathrm{Ni}$. Theoretical calculations predicted the first excited state energy above $2 \mathrm{MeV}[5,6]$, which would also be consistent with a doubly magic character.

Only recently in-beam $\gamma$-ray spectroscopy of the elusive ${ }^{78} \mathrm{Ni}$ was performed [7]. The experimental results together with theoretical calculations [7] confirm the magic nature of ${ }^{78} \mathrm{Ni}$ but suggest competing spherical and deformed configurations in the region, and predict the breakdown of the $Z=28$ shell closure toward heavier nickel isotopes. In this context, mapping the $Z=28$ isotopes and the $N=50$ isotones is of great interest. Monopole drifts have been observed in neighboring $Z=29 \mathrm{Cu}$ isotopes leading to the modification of ground-state configurations [8,9], which may also point to a weakening of the $Z=28$ gap.

The strength of the $N=50$ neutron shell gap and the proton structure close to ${ }^{78} \mathrm{Ni}$ can be obtained from the $N=50$ isotones, and in particular from the odd-proton neighbors ${ }^{79} \mathrm{Cu}$ and ${ }^{81} \mathrm{Ga}$. The nucleus ${ }^{79} \mathrm{Cu}$ was not reachable until very recently, when the first spectroscopic study was reported [10] and its mass was precisely measured [11]. From these studies the magicity of ${ }^{78} \mathrm{Ni}$ and the persistence of the $Z=28$ gap is confirmed. In this way ${ }^{79} \mathrm{Cu}$ can be described as a valence proton coupled to the ${ }^{78} \mathrm{Ni}$ core. A spin parity of $5 / 2^{-}$is suggested for its ground state (gs), while the $3 / 2^{-}$ first-excited state is proposed at a high energy of $656 \mathrm{keV}$ [10]. The lowering of the $5 / 2^{-}$state and eventual inversion with the $3 / 2^{-}$is shown for the $\mathrm{Cu}$ isotopic chain by recent Monte Carlo shell-model calculations [9]. The ${ }^{79} \mathrm{Cu}$ results are consistent with the description of ${ }^{80} \mathrm{Zn}$, two protons above ${ }^{78} \mathrm{Ni}$, in terms of two-proton configurations on top of the ${ }^{78} \mathrm{Ni}$ core [12], which also confirm the persistence of the $N=50$ shell closure.

The next odd $N=50$ isotope, ${ }^{81} \mathrm{Ga}$, is the subject of this paper. With three protons outside ${ }^{78} \mathrm{Ni}$, it provides important information about proton single-particle configurations and on the strength of the $N=50$ shell closure when the number of protons increase. In our study we have produced ${ }^{81} \mathrm{Zn}$ isotopes at ISOLDE, CERN to populate ${ }^{81} \mathrm{Ga}$ in $\beta^{-}$ decay. We have used $\gamma$-ray spectroscopy to greatly extend the known level scheme, and the Advanced Time-Delayed ("fasttiming") $\beta \gamma \gamma(\mathrm{t})$ method $[13,14]$ to measure excited level lifetimes, and deduce transition probabilities, which provide more stringent tests of the theoretical models and will help interpret the structure of ${ }^{81} \mathrm{Ga}$.

\section{PREVIOUS KNOWLEDGE OF THE ${ }^{81} \mathrm{Ga}$}

The first studies of the decay of ${ }^{81} \mathrm{Zn}$ were performed in 1991 at the ISOLDE CERN facility by Kratz et al. [15]. The half-life and the $\beta$-delayed neutron emission probability were investigated, the reported values being $T_{1 / 2}=290(50) \mathrm{ms}$ and $P_{n}=7.5(30) \%$. Later, two $\gamma$ transitions of 351 and $452 \mathrm{keV}$ were identified as belonging to the decay of ${ }^{81} \mathrm{Zn}$ to ${ }^{81} \mathrm{Ga}$ by Verney et al. [16] at PARRNe and by Köster et al. [17] at ISOLDE. In the latter measurement, due to the notable ${ }^{80} \mathrm{Ga}$ activity present in the decay of ${ }^{81} \mathrm{Zn}$, a lower limit of $10 \%$ for the $P_{n}$ value was suggested. Theoretical calculations [18] predicted $P_{n}=13 \%$. Measurements performed at the NSCL and published in 2010 by Hosmer et al. [3] proposed a considerably longer half-life of $474_{-83}^{+93} \mathrm{~ms}$ and a higher $P_{n}=30(13) \%$ value for ${ }^{81} \mathrm{Zn}$.

The $\beta^{-}$decay of ${ }^{81} \mathrm{Zn}$ was studied again at the PARRNe mass separator in Ref. [19]. The statistics obtained in this experiment allowed for the $351.1-\mathrm{keV}$ transition to be attributed to ${ }^{81} \mathrm{Ga}$ due to the new ${ }^{81} \mathrm{Zn}$ half-life value of $391(65) \mathrm{ms}$. The existence of the second excited state at $802.8 \mathrm{keV}$ was confirmed by the observation of a $451.7-\mathrm{keV} \gamma$ ray in coincidence with the $351.1-\mathrm{keV}$ line. The first excited state was defined by the $351.1-\mathrm{keV}$ transition based on $\gamma$-intensity considerations. A third, weak transition was detected at $1621.6 \mathrm{keV}$ and a tentative state of the same energy was added to the level scheme. Spin assignments for the ground, first-excited, and second-excited states of ${ }^{81} \mathrm{Ga}$ were tentatively proposed to be $\left(5 / 2^{-}\right),\left(3 / 2^{-}\right)$, and $\left(3 / 2^{-}\right)$, respectively, based on shell-model calculations and proton single-particle states. The authors suggested $\left(1 / 2^{+}\right)$spin parity for the ${ }^{81} \mathrm{Zn}$ ground state. Magnetic-moment measurements performed at ISOLDE [20] yielded a ${ }^{81} \mathrm{Ga}$ ground-state spin-parity value of $5 / 2^{-}$, confirming the earlier tentative assignment.

A study of the ${ }^{81} \mathrm{Ga}$ structure was performed at LNL via heavy-ion multinucleon transfer [21]. Several $\gamma$ rays were attributed to ${ }^{81} \mathrm{Ga}$, and specifically a $1236-\mathrm{keV}$ transition connecting a state of the same energy to the ground state, which were assigned $\left(9 / 2^{-}\right)$and $\left(5 / 2^{-}\right)$spin parities, respectively. A new measurement of the yrast states of ${ }^{81} \mathrm{Ga}$ populated in fission [22] contradicts this assignment, since none of the $\gamma$ rays reported in Ref. [21] could be confirmed. Instead, the $\left(9 / 2^{-}\right)$is observed at $1340.7 \mathrm{keV}$. The $1398.5-\mathrm{keV}$ and 1952.2-keV levels are assigned $\left(7 / 2^{-}\right)$and $\left(11 / 2^{-}\right)$spin parity, respectively [22]. The two later states are also observed by in-beam spectroscopy in knockout reactions at RIBF [23]. In spite of the large spin difference, the indirect population of these higher spin states in ${ }^{81} \mathrm{Ga}$ from the $\beta$ decay of $\left(1 / 2^{+}\right)$ ${ }^{81} \mathrm{Zn}$ ground state should be possible.

The most recent data of the $\beta$ decay of ${ }^{81} \mathrm{Zn}$ comes from HRIBF at ORNL. The results were published in 2010 by Padgett et al. [24]. The decay scheme showed six new energy levels and nine new $\gamma$ transitions in addition to the previously available ones. Transitions of $451,916,1107,1585$, and $2358 \mathrm{keV}$ were observed in coincidence with the strongest $351-\mathrm{keV}$ peak. Four other $\gamma$ rays, only observed in the singles $\gamma$ spectrum, with energies of 1458, 1936, 4294, and $4880 \mathrm{keV}$ were placed directly feeding the ground state. A new value of 304(13) $\mathrm{ms}$ for the ${ }^{81} \mathrm{Zn}$ half-life was established and a $\beta$-delayed neutron branch of $12(4) \%$ was determined using the $1083-\mathrm{keV}$ transition in ${ }^{80} \mathrm{Ge}$. In this work, a spinparity assignment of $\left(5 / 2^{+}\right)$for the ground state of ${ }^{81} \mathrm{Zn}$, different from the earlier value, was proposed, based on the 
$J^{\pi}=5 / 2^{-81} \mathrm{Ga}$ ground state [20] and the $\beta$-feeding pattern. The first and the second excited states both received a tentative $J^{\pi}=\left(3 / 2^{-}\right)$assignment.

From the existing works, the structure of ${ }^{81} \mathrm{Ga}$ is interpreted as arising from the coupling of valence protons in the $f p$ shell, leading to negative-parity states at low excitation energy. Positive-parity states, requiring the excitation of a proton to the $g_{9 / 2}$ orbit across $Z=40$, or particle-hole excitations of the ${ }^{78} \mathrm{Ni}$ core, appear at energies above $4 \mathrm{MeV}$. The high excitation energy points toward a robust $N=50$ neutron shell closure, in agreement with the recently observed ${ }^{79} \mathrm{Cu}$ structure [10].

\section{EXPERIMENTAL SETUP AND DATA ANALYSIS}

The present experiment was performed at the ISOLDE CERN facility in the framework of a systematic fast-timing investigation of neutron-rich nuclei populated following the decay of $\mathrm{Zn}$ isotopes [25-27]. The selectivity and efficiency for the production of $\mathrm{Zn}$ ion beams had been previously optimized [28] in order to enhance the beam purity for ${ }^{77-82} \mathrm{Zn}$ ions. Proton pulses with an average charge of $5 \mu \mathrm{C}$ and 1.4-GeV energy, coming from the PS-Booster in intervals of multiples of $1.2 \mathrm{~s}$, were converted into fast neutrons [29] that impinged onto a hot $\sim 2000^{\circ} \mathrm{C} \mathrm{UC}_{2}$ /graphite target, inducing fission reactions. The thermally extracted products were guided through a temperature-controlled quartz glass transfer line [30] into a $\mathrm{W}$ ionizer where selective ionization was performed by the ISOLDE Resonance Ionization Laser Ion Source [31]. The single-charged $A=81$ ions were mass separated by the magnetic high-resolution mass separator, accelerated to $60 \mathrm{keV}$, and directed to the experimental setup.

The mass-separated ${ }^{81} \mathrm{Zn}$ nuclei were continuously collected on an aluminum stopper foil, creating a saturated source. The estimated yield of ${ }^{81} \mathrm{Zn}$ was 600 ions $/ \mu \mathrm{C}$. Since $\mathrm{Rb}$ atoms partially survived the quartz transfer line selection and were surface ionized on the walls of the ionizer, the longlived $T_{1 / 2}=4.57 \mathrm{~h}$ contaminant ${ }^{81} \mathrm{Rb}$ was present in the beam, with about five times higher production than ${ }^{81} \mathrm{Zn}$, but much lower activity during the data taking. An electrostatic deflector (beam gate) blocking the delivery of ions to the experimental station, was used to avoid the accumulation of long-lived ${ }^{81} \mathrm{Rb}$ activity coming from the target long after most of the ${ }^{81} \mathrm{Zn}$ had been released. For the mass 81 experiment, the beam gate was closed $600 \mathrm{~ms}$ after proton impact, and the collected species were allowed to decay out.

The experimental setup included two HPGe detectors, two $\mathrm{LaBr}_{3}(\mathrm{Ce})$ detectors, and an NE111A plastic scintillator for $\beta$-particle detection, very close to the beam deposition point. In particular, the 3-mm-thick plastic scintillator was located less than $1 \mathrm{~mm}$ away from the stopper foil in order to maximize the detection efficiency. This thin detector assures ultrafast and uniform time response independent of the incident $\beta$ energy. The germanium detectors were used for the detection of $\gamma$ radiation in the range of 30 to $7000 \mathrm{keV}$; their energy resolution was $2.0 \mathrm{keV}$ at ${ }^{60} \mathrm{Co}$ energies. Coincidences with the $\beta$ detector were used for $\gamma$-ray background suppression and $\gamma-\gamma$ coincidences between the HPGe detectors to determine the decay scheme. For the lifetime measurements of the excited states in the tens of picoseconds to nanosecond range, fast-response inorganic $\mathrm{LaBr}_{3}(\mathrm{Ce})$ crystals with the shape of truncated cones [32] were mounted almost perpendicularly to the germanium detectors. These scintillator crystals have a fast-decay component that makes it possible to achieve very good time resolution while maintaining acceptable energy resolution [32,33]. Each crystal was mounted onto a Photonis XP20D0 fast-response 2-inch photomultiplier tube (PMT), optimized to give fast time response at the cost of lower gain.

The signals from all the detectors were processed by a digital data acquisition (DAQ) system composed of four Pixie4 Digital Gamma Finder cards, specially designed for $\gamma$-ray spectroscopy [34]. For the energy analysis, the HPGe signals from the preamplifier were fed into the DAQ, while the much faster scintillator signals taken from the last PMT dynodes were shaped before they were sent to the digital system. The PMT anode signals from the scintillator detectors were used for fast timing. The signals were processed by analog constant fraction discriminators and then sent to time-to-amplitude converter (TAC) modules to measure the time difference between the $\beta$ start detector and the two $\gamma$ scintillators, which acted as stop detectors. Additionally, two more TACs were included to record time differences between the fast $\beta$ and the slower HPGe detectors. Logic signals related to the beam parameters were also recorded including the time of proton impact on target which triggers the production and release of $\mathrm{Zn}$ ions out of the target. These triggered beam pulses define the starting time for the ${ }^{81} \mathrm{Zn}$ accumulation and were used to rule out the long half-life contaminants by setting time gates with this signal as a reference. The Pixie-4 system is configured to write data in a triggerless mode. Coincident events were constructed off-line in order to correlate the time differences, the detector energies, and the other relevant running parameters.

For the data analysis, a time gate starting $50 \mathrm{~ms}$ after the proton impact and ending $1200 \mathrm{~ms}$ after it was adopted, which minimizes the presence of long-lived daughter activity in the $A=81$ data. Coincidence with $\beta$ particles was imposed to suppress the background contributions. The energy spectra contain $\gamma$ lines from the ${ }^{81} \mathrm{Zn}$ decay chain and also a negligible fraction of contaminant lines from the $\beta^{+}$decay of ${ }^{81} \mathrm{Rb}$ to ${ }^{81} \mathrm{Kr}$. The strongest line of this decay $(446 \mathrm{keV})$ was around $4 \%$ as intense as the $351-\mathrm{keV}$ transition of ${ }^{81} \mathrm{Ga}$. In addition, the subtraction of the long-lived activity (using a delayed time window after proton impact) provides a clean energy spectrum containing $\gamma$ rays from the $\beta$ decay of ${ }^{81} \mathrm{Zn}$, including the $\beta$-delayed neutron emission branch. The $\gamma$ rays from the decay can be assigned to deexcite energy levels in the ${ }^{81} \mathrm{Ga}$ and ${ }^{80} \mathrm{Ga}$ nuclei and their daughters.

In the first $\sim 50 \mathrm{~ms}$ after proton impact on the target, neutron-capture $\gamma$ rays are observed in the HPGe spectra. This is due to neutrons that escape the converter in the target area, thermalize, and reach the measurement station. These capture lines were used for high-energy calibration of the HPGe detectors up to $7 \mathrm{MeV}$, together with sources of ${ }^{133} \mathrm{Ba},{ }^{138} \mathrm{Cs}$, ${ }^{140} \mathrm{Ba}$, and ${ }^{152} \mathrm{Eu}$ for the energy and efficiency calibrations.

Excited-state lifetimes have been measured using the Advanced Time-Delayed $\beta \gamma \gamma(\mathrm{t})$ fast-timing method [13,14,35]. Coincidences between the fast-response plastic scintillator 


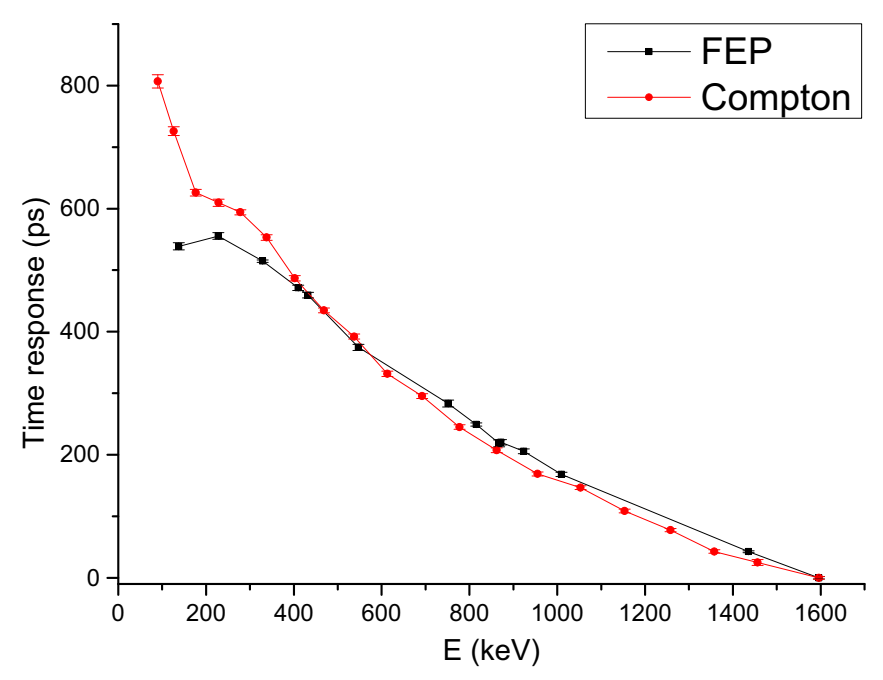

FIG. 1. Relative Compton response (red) and FEP prompt curve (black) of one of the $\mathrm{LaBr}_{3}(\mathrm{Ce})$ detectors used in our experimental setup. The calibration is obtained with an $A=140$ source.

and the $\mathrm{LaBr}_{3}(\mathrm{Ce})$ crystals were used. The method consists in the use of triple $\beta \gamma \gamma$ coincident events. The $\beta$-HPGeHPGe coincidences allow the decay branches to be identified whereas the $\beta$-HPGe- $\mathrm{LaBr}_{3}(\mathrm{Ce})$ events make it possible to measure the lifetimes down to the tens-of-picoseconds range. The decay path is selected with a gate on the HPGe detector, whereas the lifetime is obtained from the time difference between the $\beta$ plastic scintillator and the $\mathrm{LaBr}_{3}(\mathrm{Ce}) \gamma$ signal, which start and stop a TAC, respectively. With a full width at half maximum (FWHM) time resolution of the $\mathrm{LaBr}_{3}(\mathrm{Ce})$ detectors of $110 \mathrm{ps}$ for the ${ }^{60} \mathrm{Co}$ full energy peaks [32] and the very fast time response of the $\beta$ plastic scintillator below $50 \mathrm{ps}$, the $\beta \mathrm{LaBr}_{3}(\mathrm{Ce})$ time distribution for prompt transitions, typically quasi-Gaussian, has a FWHM of 120 ps. Half-lives longer than about 60 ps will appear as a slope on the delayed part of the time spectrum. The lifetime can be extracted by the deconvolution of the slope of the time spectrum from the prompt time distribution. Shorter halflives, down to tens of ps, are obtained by the centroid shift of the time distribution with respect to the time distribution of a prompt transition of the same energy [13].

The application of the centroid shift method requires the use of calibration curves for the time response as a function of energy for the full-energy peaks (FEP) and Compton events. For the FEP prompt response curve we have used peaks from a ${ }^{140} \mathrm{Ba} /{ }^{140} \mathrm{La}$ calibration source, primarily from excited states of ${ }^{140} \mathrm{Ce}$ with known half-lives [36], including both the correction by the Compton curve and the level lifetime. Both curves are plotted in Fig. 1. The Compton response curve has been constructed with the time response of Compton events arising from the $1596-\mathrm{keV} \gamma$ transition from ${ }^{140} \mathrm{Ce}$. The time response curves have a smooth behavior versus energy, and they are very similar for $\gamma$-ray energies above $400 \mathrm{keV}$. At lower energies the curves differ due to the physics of the interaction [35], especially in the region of backscatter and $\mathrm{X}$-ray events.

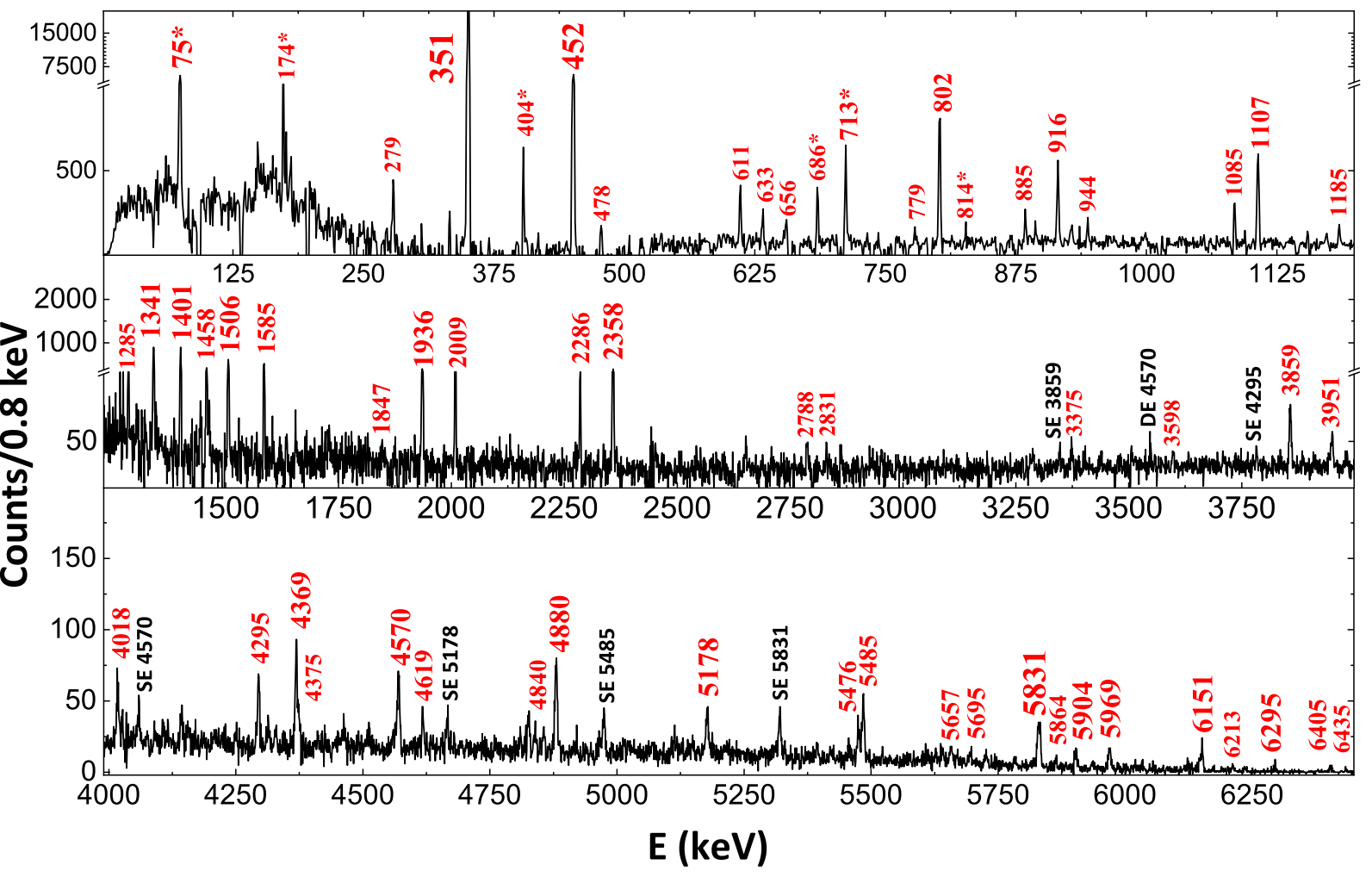

FIG. 2. The $\beta$-gated $\gamma$-ray singles spectrum obtained following the decay of ${ }^{81} \mathrm{Zn}$, after subtraction of the long-lived activity. The transitions in ${ }^{81} \mathrm{Ga}$ are labeled by their energies. Some transitions from the $\beta^{-} \mathrm{n}$ decay of ${ }^{81} \mathrm{Zn}$ to ${ }^{80} \mathrm{Ga}$ are marked with asterisks. 
Peak and background centroid corrections are made separately following their respective walk curves. Normally the FEPs sit on background arising mainly from Compton events coming from transitions with higher energies. The time delay originating from the background component is corrected according to the peak-to-background ratio with the help of the Compton correction curve [35]. The resulting centroid of the FEP time distribution is then compared with the baseline given by the FEP correction curve. Any delay relative to the curve is then due to the lifetime carried by the transition giving rise to the FEP and can be related to levels in the nuclide of interest. In addition, the timing analysis includes standard corrections for the very small dependence of the $\beta$ time response with energy, and, if needed, for small electronics drifts during the measurement.

\section{RESULTS}

The significantly higher statistics obtained in our experiment compared to previous works is illustrated by the spectrum shown in Fig. 2. Transitions up to $6.5 \mathrm{MeV}$ in energy are observed, along with the strongest transitions at 351.1 and $451.6 \mathrm{keV}$. More than $20 \gamma$ decays with sizable intensity are detected beyond $4 \mathrm{MeV}$.

Transitions arising from the $\beta^{-}$decay of ${ }^{81} \mathrm{Zn}$ have been identified from their time spectra after proton impact, which is consistent with the ${ }^{81} \mathrm{Zn}$ half-life of $0.32(5) \mathrm{s}$ adopted in Ref. [37]. In our experiment, the ${ }^{81} \mathrm{Zn}$ half-life has been measured using the time spectrum gated directly on three of the strongest ${ }^{81} \mathrm{Ga}$ transitions of 351,452 , and $1341 \mathrm{keV}$ [see Fig. 3(a)]. A simple exponential decay plus constant background function has been used in the time range from $700 \mathrm{~ms}$ (with a slight delay after the end of implantation) to $2400 \mathrm{~ms}$, restricting the time between proton impact on target to two or more cycles (2.4 s or longer). The weighted mean value obtained yields $T_{1 / 2}=290(4) \mathrm{ms}$, in agreement with the recent literature values $[4,24]$.

To obtain the ${ }^{81} \mathrm{Ga}$ half-life, the $216-$ and $828-\mathrm{keV}$ transitions in ${ }^{81} \mathrm{Ge}$ [38] were used. An exponential fit was employed by limiting the lower time boundary to $2000 \mathrm{~ms}$ after proton impact, which corresponds to 6.9 half-lives of ${ }^{81} \mathrm{Zn}$, when less than $1 \%$ remains. The fitted slope leads to $T_{1 / 2}=1.25(3) \mathrm{s}$ for ${ }^{81} \mathrm{Ga}$ as depicted in Fig. 3, consistent with the literature value of $1.217(5) \mathrm{s}$ [37].

Finally, gating on the $659-\mathrm{keV}$ transition which deexcites the $2^{+} 659-\mathrm{keV}$ level in ${ }^{80} \mathrm{Ge}$, we get the apparent ${ }^{80} \mathrm{Ga}$ half-life, where a $22-\mathrm{keV}^{-}$isomer has been identified above the $6^{-}$ground state [25]. The half-lives of these states were previously measured as 1.3(2) and 1.9(1) s, respectively, in the $\beta$-decay experiment described in Ref. [39]. According to the level scheme from Fig. 5 of Ref. [39], the $659-\mathrm{keV}$ state is $\beta$-fed directly from the low-spin isomer while the high-spin isomer populates it via the $1083-\mathrm{keV} \gamma$ ray that deexcites the $1743-\mathrm{keV}$ level. Therefore, the time since proton impact spectrum gated by the $659-\mathrm{keV}$ will contain the contribution of half-lives from both isomers and the fitted value should lie between 1.3 and $1.9 \mathrm{~s}$. Using the same time fitting conditions as before we get $T_{1 / 2}=1.70(3) \mathrm{s}$. As discussed in Sec. IV B below, this value is mainly due to the $3^{-}$isomer half-life,

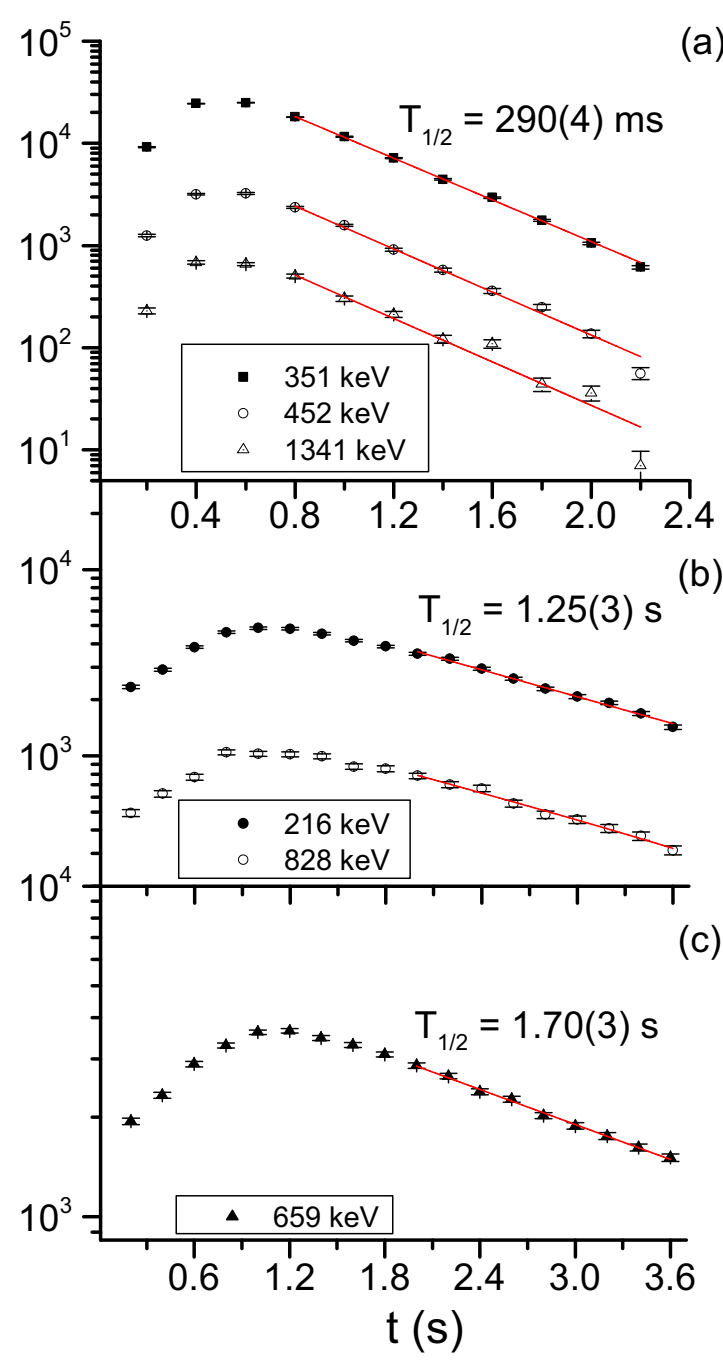

FIG. 3. Ground-state half-lives measured in this work. (a) ${ }^{81} \mathrm{Zn}$ half-life obtained from three of the strongest ${ }^{81} \mathrm{Ga}$ transitions at 351 , 452, and $1341 \mathrm{keV}$. (b) Measurement of the ${ }^{81} \mathrm{Ga}$ half-life by gating on the 216- and 828-keV transitions in ${ }^{81} \mathrm{Ge}$. (c) Apparent ${ }^{80} \mathrm{Ga}$ halflife (combined ground state and $22-\mathrm{keV}$ isomer).

which is the state predominantly populated in the $\beta$-n decay of ${ }^{81} \mathrm{Zn}$.

\section{A. ${ }^{81} \mathrm{Zn} \beta^{-}$decay to ${ }^{81} \mathrm{Ga}$}

The decay scheme of ${ }^{81} \mathrm{Ga}$ has been extended using coincidences with previously known transitions employing the $\gamma-\gamma$ coincidence spectrum between both HPGe detectors. Figure 4 shows the energy spectra in coincidence with the 351- and 2358-keV transitions. Note that $\gamma$ rays up to $5 \mathrm{MeV}$ are registered in coincidence with the strong $351-\mathrm{keV}^{81} \mathrm{Ga}$ transition. Table I summarizes the information about the $\gamma$ transitions associated with the decay of ${ }^{81} \mathrm{Zn}$ to ${ }^{81} \mathrm{Ga}$. The relative intensities of the $\gamma$-ray transitions were extracted using the full-energy peak areas from the $\beta$-gated $\gamma$-ray spectrum and were normalized to the strongest transition at $351 \mathrm{keV}$.

Based on the $\gamma-\gamma$ coincidences, 70 transitions that were not previously observed in Ref. [24] have been placed in the level 


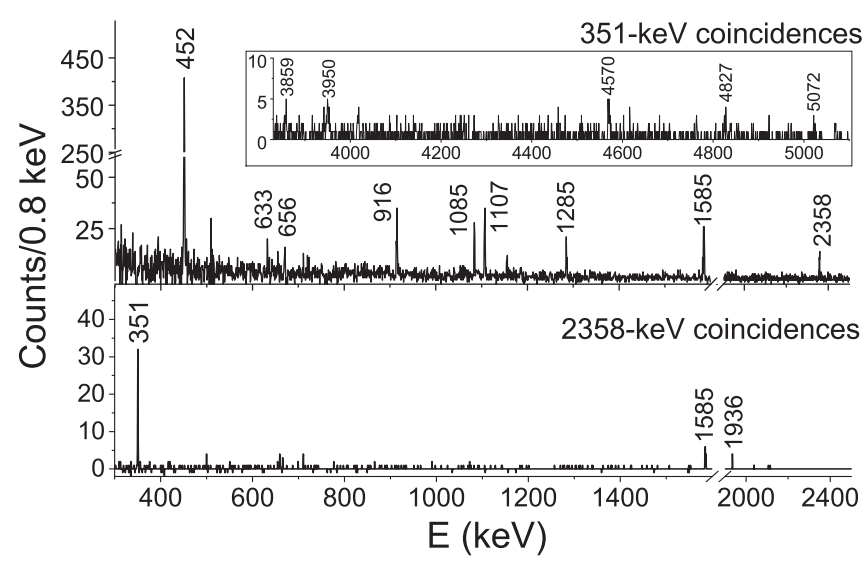

FIG. 4. $\gamma-\gamma$ coincidence spectra gated by the strongest, $351-\mathrm{keV}$ transition (top) and the 2358-keV line (bottom).

scheme, which is shown in Figs. 5 and 6. Weak transitions that were not observed in coincidence with strong ${ }^{81} \mathrm{Ga} \gamma$ rays have not been included, since they could also belong to the level scheme of ${ }^{80} \mathrm{Ga}$ populated in the $\beta^{-} \mathrm{n}$ decay of ${ }^{81} \mathrm{Zn}$ (see Sec. IV D). Such is the case of 279-, 505-, 627-, 779-, 2627-, and 2943-keV $\gamma$ rays. Their combined intensities amount to $1.1 \%$ of the total $\gamma$ intensity. However, some of the weak $\gamma$ rays of $478,656,894$, and $1185 \mathrm{keV}$, fit the energy differences between already established levels and were tentatively placed in the level scheme. They are marked with broken lines. The high-energy $\gamma$ rays not observed in coincidence with those at 351 and $452 \mathrm{keV}$ were placed as deexciting a state with the same energy. We note that the available energy window for $\beta^{-}$decay is $Q_{\beta^{-}}=11428(6) \mathrm{keV}$ [40], compared to a value of $Q_{\beta^{-} n}=4953(6) \mathrm{keV}$ [40] for $\beta$-delayed neutron emission. Therefore, $\gamma$ rays with energies above $5 \mathrm{MeV}$ that follow the ${ }^{81} \mathrm{Zn}$ half-life must belong to ${ }^{81} \mathrm{Ga}$ and not to ${ }^{80} \mathrm{Ga}$.

In this way, 47 excited states of ${ }^{81} \mathrm{Ga}$ in the energy range up to the neutron separation energy of 6476(4) $\mathrm{keV}$ [40] have been observed, 40 of them for the first time. We confirm the existence of 351.1-, 802.5-, 1266.7-, 1458.3-, 1936.4-, 4294.9-, and 4880.4-keV levels, already seen in the latest $\beta$-decay study [24]. The states identified as $\left(9 / 2^{-}\right)$and $\left(11 / 2^{-}\right)$in fission $\gamma$-ray spectroscopy [22] are also observed at 1341.0 and $1952.4 \mathrm{keV}$ [26].

\section{B. $\beta$-delayed neutron emission probability of ${ }^{81} \mathbf{Z n}$}

To obtain the $\beta$-delayed neutron emission probability of ${ }^{81} \mathrm{Zn}$ we compared the number of decays arising from the direct ${ }^{81} \mathrm{Zn} \beta$-decay chain, using the absolute intensities of

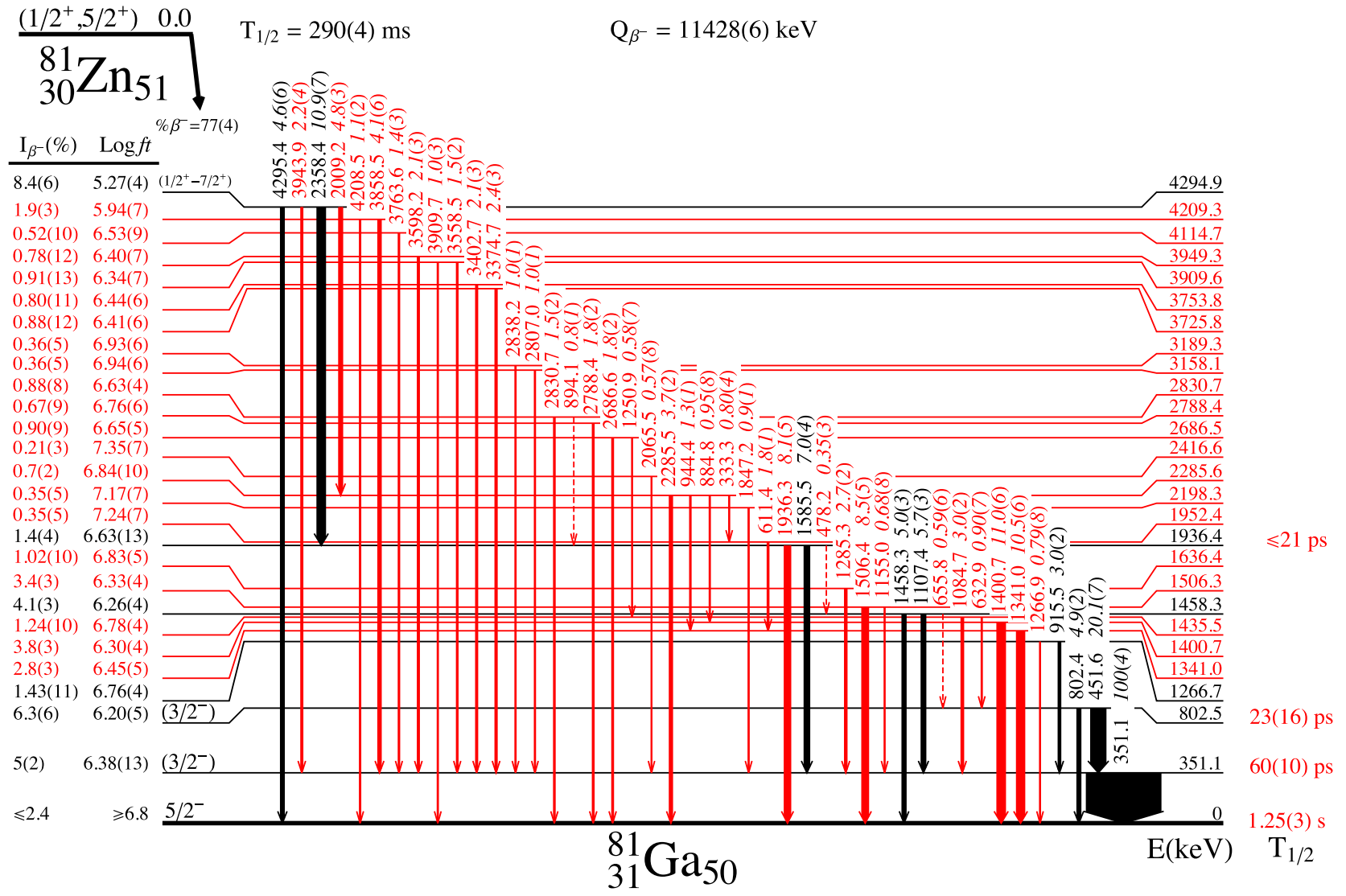

FIG. 5. Level scheme of ${ }^{81} \mathrm{Ga}$ up to $4.3 \mathrm{MeV}$ in energy populated following the $\beta$ decay of ${ }^{81} \mathrm{Zn}$. Dashed arrows indicate tentatively placed transitions. For the sake of clarity, the decay scheme has been split in two sections. 


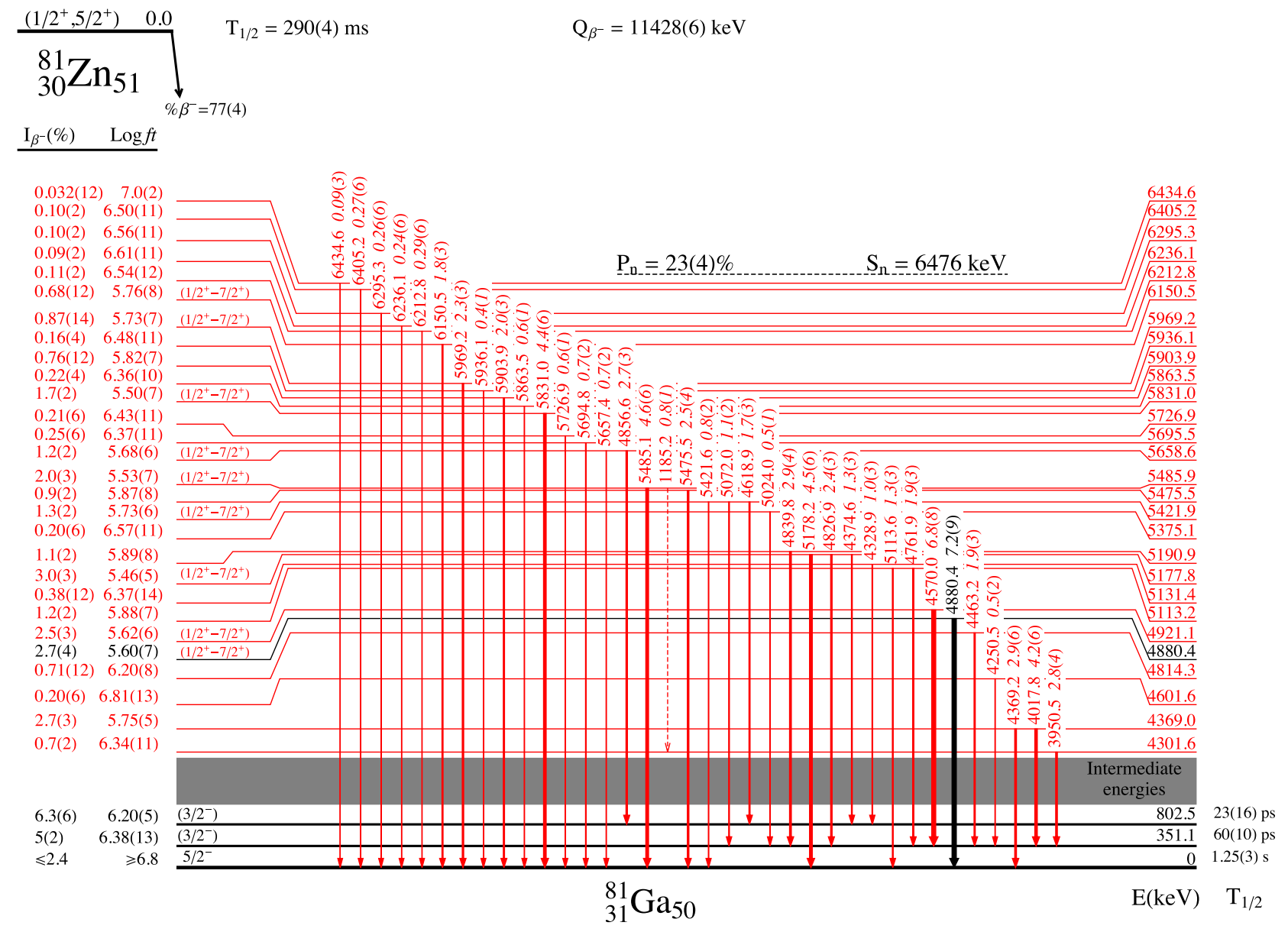

FIG. 6. Level scheme of ${ }^{81} \mathrm{Ga}$ populated in the $\beta$ decay of ${ }^{81} \mathrm{Zn}$, containing the high-lying states between 4.3 and $6.5 \mathrm{MeV}$ in energy. Dashed arrows indicate tentatively placed transitions.

the two strongest lines in ${ }^{81} \mathrm{Ge}$, at 216 and $828 \mathrm{keV}$ [37], to the ${ }^{81} \mathrm{Zn} \beta^{-} \mathrm{n}$ decay branch of the $A=80$ chain, taking the absolute intensities per 100 parent decays of 666-, 1207-, and $1645-\mathrm{keV}$ lines from the $\beta$ decay of ${ }^{80} \mathrm{As}$ to ${ }^{80} \mathrm{Se}[41,42]$. We employ the literature value of $11.9(7) \%$ for the ${ }^{81} \mathrm{Ga} \beta^{-} \mathrm{n}$ branch [37] and apply a small correction factor coming from the ${ }^{80} \mathrm{Ga} \beta^{-} \mathrm{n}$ decay probability of $0.86(7) \%$, also taken from the literature [43]. Determining the areas of the above mentioned transitions directly from the $\beta$-gated singles spectrum and taking into account the absolute intensities we obtain $P_{n}=23(4) \%$ for ${ }^{81} \mathrm{Zn}$.

\section{Direct $\beta$ feeding to the ${ }^{81}$ Ga ground state}

For the absolute $\beta$ feeding to be derived, it is necessary to obtain the ground-state $\beta$ feeding. Since there is no isomeric state reported for ${ }^{81} \mathrm{Ga}$, the total ground state feeding, both $\gamma$ and $\beta$, proceeds through the ${ }^{81}$ Ga ground-state $\beta$ decay to states in ${ }^{81} \mathrm{Ge}$, and via the $\beta$-delayed neutron emission branch to states in ${ }^{80} \mathrm{Ge}$. A $\beta$-decaying isomer exists in ${ }^{81} \mathrm{Ge}$ at 679 $\mathrm{keV}$ [38], for which no $\gamma$-ray branch was observed. Therefore, these two states need to be considered in the $\beta$ decay of ${ }^{81} \mathrm{Ga}$, both for $\gamma$ and $\beta$ feeding. For the $\beta$-n branch from ${ }^{81} \mathrm{Ga}$ we take an adopted $P_{n}$ value of $11.9(7) \%$ from Ref. [37]. In addition, for the ${ }^{81} \mathrm{Zn}$ the $P_{n}$ value of 23(4)\% from our data are used, as described in Sec. IV B above.

The $\gamma$-ray intensities in ${ }^{81} \mathrm{Ga}$ and ${ }^{81} \mathrm{Ge}$ are obtained from our data without time conditions, thus containing the shortlived and long-lived decay products from ${ }^{81} \mathrm{Zn}$ and its daughters, and normalized to the strongest $351-\mathrm{keV}$ transition in ${ }^{81} \mathrm{Ga}$, Table I. The total $\gamma$ intensity feeding the ground state of ${ }^{81} \mathrm{Ga}$ is measured to be $I_{\gamma, \mathrm{gs}}^{\mathrm{Ga}}=203(4)$. In the decay of ${ }^{81} \mathrm{Ga}$, the $\gamma$-ray intensity that feeds directly the $679-\mathrm{keV}$ isomer state and the ground state amounts to 101(3) and 95(3) in the same units, respectively, and thus the $\gamma$-ray feeding both states is 196(4) units.

To estimate the $\beta$-feeding intensity to the ground state and $679-\mathrm{keV}$ isomer in ${ }^{81} \mathrm{Ge}$ we make use of the spin assignments of $9 / 2^{+}$and $1 / 2^{+}$[38]. These levels are therefore $\beta$-fed from the ${ }^{81} \mathrm{Ga} 5 / 2^{-}$ground state via first-forbidden unique $\beta$ transitions with $\Delta J=2, \Delta \pi=$ yes. It is then reasonable to consider a lower limit of $\log ^{1 U} f t=8.5$ (see Fig. 1 of Ref. [44]) for both states. The $\beta$ feeding calculated with these assumptions gives upper limits of $11.3 \%$ for the $9 / 2^{+}$ground state and $6.6 \%$ for the $679-\mathrm{keV}$ isomer represented in absolute units [5.7(56)\% and 3.3(33)\% were used for calculations]. 
TABLE I. Gamma transitions in the decay of ${ }^{81} \mathrm{Zn}$ to ${ }^{81} \mathrm{Ga}$. For those placed in the decay scheme, the initial and final level energies are given in the second and third columns. Relative intensities, normalized to 100 units for the $351-\mathrm{keV}$ transition, are provided. The strongest transitions observed in $\gamma-\gamma$ coincidences are given in the last column.

\begin{tabular}{|c|c|c|c|c|}
\hline 333.32 & 2285.61 & 1952.42 & 0.804 & $611,1341,2009$ \\
\hline 351.11 & 351.11 & 0.0 & 1004 & $\begin{array}{c}452,633,656,916,1085,1107,1155, \\
1185,1251,1285,1585,1847,2065, \\
2358,2807,2838,3375,3403,3558,3598, \\
3764,3859,3944,3950,4018,4250,4463, \\
4570,4762,4827,4840,5024,5072\end{array}$ \\
\hline $478.2^{\mathrm{b}} 2$ & 1936.41 & 1458.31 & 0.353 & \\
\hline 611.41 & 1952.42 & 1341.01 & 1.81 & 333,1341 \\
\hline 632.91 & 1435.51 & 802.51 & 0.907 & 351,452 \\
\hline $655.8^{\mathrm{b}} 2$ & 1458.31 & 802.53 & 0.596 & 351 \\
\hline 802.41 & 802.53 & 0.0 & 4.92 & \\
\hline 884.82 & 2285.61 & 1400.72 & 0.958 & 1401,2009 \\
\hline 1107.42 & 1458.31 & 351.11 & 5.73 & 351 \\
\hline 1155.02 & 1506.31 & 351.11 & 0.688 & 351 \\
\hline $1185.2^{\mathrm{b}} 2$ & 5485.93 & 4301.64 & 0.81 & 351 \\
\hline 1250.92 & 2686.52 & 1435.51 & 0.587 & 351,1085 \\
\hline 1266.96 & 1266.73 & 0.0 & 0.798 & \\
\hline 1285.31 & 1636.42 & 351.12 & 2.72 & 351 \\
\hline 1341.01 & 1341.01 & 0.0 & 10.56 & $333,611,944,2009$ \\
\hline 1400.71 & 1400.72 & 0.0 & 11.06 & 885 \\
\hline 1458.32 & 1458.31 & 0.0 & 5.03 & \\
\hline 1506.41 & 1506.31 & 0.0 & 8.55 & \\
\hline 1585.51 & 1936.41 & 351.11 & 7.04 & 351,2358 \\
\hline 2788.43 & 2788.43 & 0.0 & 1.82 & \\
\hline 2807.03 & 3158.14 & 351.11 & 1.01 & 351 \\
\hline 2830.73 & 2830.73 & 0.0 & 1.52 & \\
\hline 2838.27 & 3189.37 & 351.11 & 1.01 & 351 \\
\hline 3374.76 & 3725.86 & 351.11 & 2.43 & 351 \\
\hline 3402.74 & 3753.84 & 351.11 & 2.13 & 351 \\
\hline 3558.55 & 3909.64 & 351.11 & 1.52 & 351 \\
\hline 3598.25 & 3949.35 & 351.11 & 2.13 & 351 \\
\hline 3763.67 & 4114.77 & 351.11 & 1.43 & 351 \\
\hline 3858.54 & 4209.33 & 351.11 & 4.16 & 351 \\
\hline 3909.78 & 3909.64 & 0.0 & 1.03 & \\
\hline 3943.95 & 4294.91 & 351.11 & 2.24 & 351 \\
\hline 3950.54 & 4301.64 & 351.11 & 2.84 & 351 \\
\hline 4017.85 & 4369.04 & 351.11 & 4.26 & 351 \\
\hline 4208.56 & 4209.33 & 0.0 & 1.12 & \\
\hline 4250.55 & 4601.65 & 351.11 & 0.52 & 351 \\
\hline 4295.44 & 4294.91 & 0.0 & 4.66 & \\
\hline 4328.96 & 5131.44 & 802.51 & 1.03 & 351,452 \\
\hline
\end{tabular}


TABLE I. (Continued.)

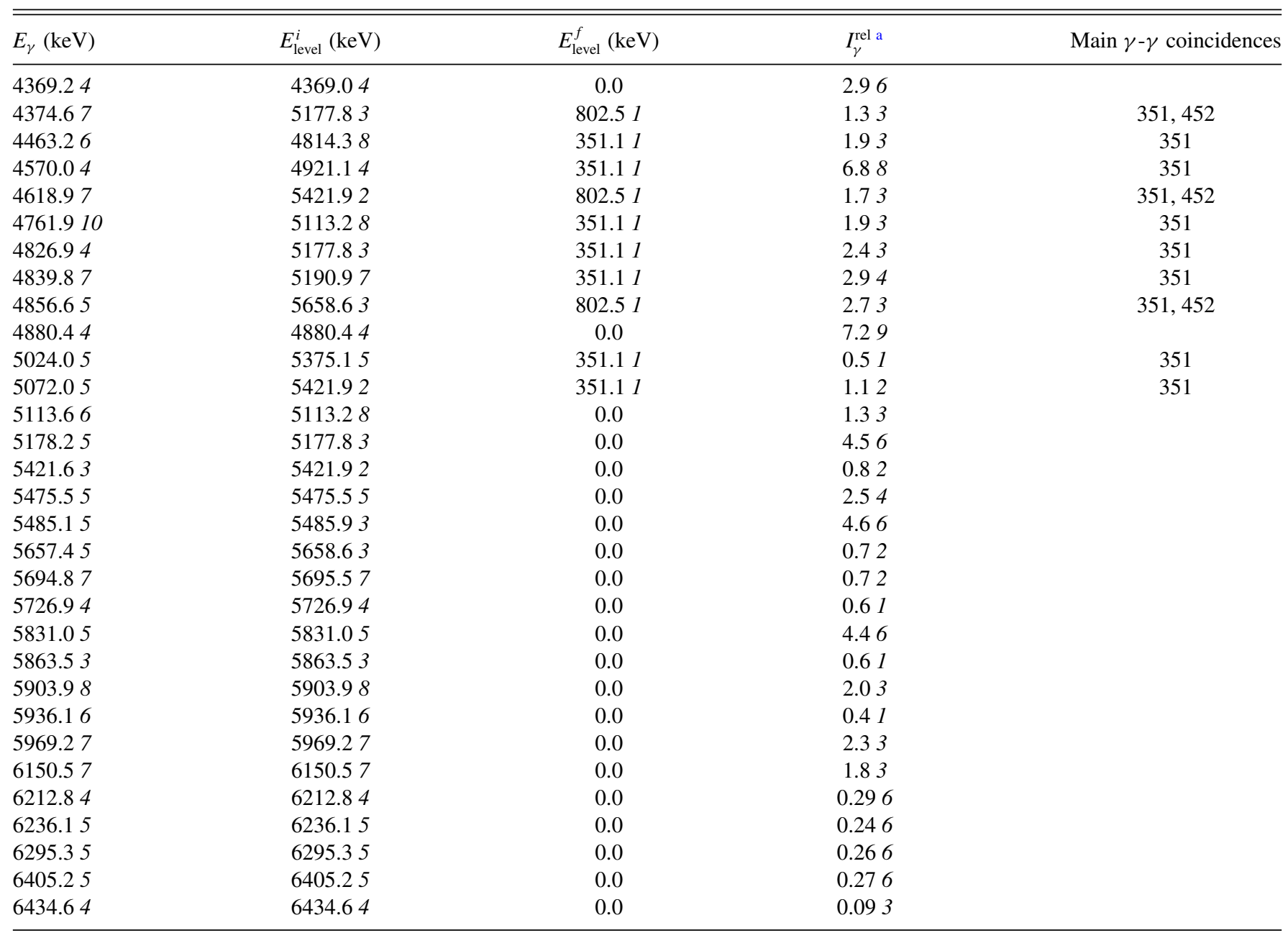

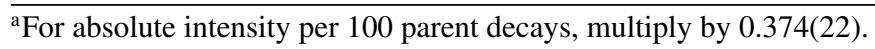

${ }^{\mathrm{b}}$ Weak transition, not observed in $\gamma-\gamma$ coincidences. Tentatively placed in the level scheme.

With these assumptions the value of the ground-state $\beta$ feeding in ${ }^{81} \mathrm{Ga}$ is extracted from the intensity balance and is given with an upper limit of $2.4 \%$. This leads to $\log f t \geqslant 6.8$, in good agreement with the systematics and selection rules for the first-forbidden nonunique $\beta$ decay transitions in the region.

Using this value, the apparent $\beta$ feeding of the remaining levels, $I_{\beta}(E)$, is obtained by the intensity balance between feeding and deexciting $\gamma$ rays. Internal conversion is neglected. High-energy transitions could have been missed or misplaced if coincidences are not observed, which means that the $\beta$ feeding would be slightly modified. We note the small energy gap between the highest level at $6434.6 \mathrm{keV}$ and the neutron separation energy, $S_{n}=6476(4) \mathrm{keV}$, which is still far from the available $\beta$-decay window, $Q_{\beta^{-}}=11428(6) \mathrm{keV}$ [40]. With the ${ }^{81} \mathrm{Zn} \beta$-delayed neutron emission probability $P_{n}=23(4) \%$ and the ground-state feeding [taking $I_{\beta, \mathrm{gs}}^{\mathrm{Ga}}=1.2(12) \%$ ], an absolute normalization factor of $0.374(22)$ is obtained for the $\gamma$ intensities in the decay of ${ }^{81} \mathrm{Zn}$ to ${ }^{81} \mathrm{Ga}$ from the relative ones tabulated in Table I.

\section{D. ${ }^{81} \mathrm{Zn} \beta^{-}$n decay to ${ }^{80} \mathrm{Ga}$}

As discussed above, $\beta$-delayed neutron emission is energetically allowed for the decay of ${ }^{81} \mathrm{Zn}$, with a ${ }^{81} \mathrm{Ga}$ neutron separation energy $S_{n}=6476(4) \mathrm{keV}$ [40], well within the $Q_{\beta^{-}}$ window. The analysis of the $\beta$-gated $\gamma$ spectrum has allowed $11 \gamma$ transitions to be assigned to ${ }^{80} \mathrm{Ga}$ populated following the $\beta^{-} \mathrm{n}$ decay of ${ }^{81} \mathrm{Zn}$. The nuclide ${ }^{80} \mathrm{Ga}$ was studied at ISOLDE during the same experimental run, populated in the $\beta^{-}$decay of ${ }^{80} \mathrm{Zn}$, and the results of the analysis were published by Lică et al. [25]. The $\gamma-\gamma$ coincidence analysis provides information to place the observed ${ }^{80} \mathrm{Ga}$ transitions deexciting nine previously known low-energy levels. Our new scheme of ${ }^{80} \mathrm{Ga}$ from $\beta^{-} \mathrm{n}$ decay of ${ }^{81} \mathrm{Zn}$ plotted in Fig. 7 is consistent with the structure from Ref. [25]. Table II contains the detailed information about the $\gamma$ transitions and fed energy levels. We neglect any direct feeding of the $6^{-}$ground and the $3^{-}$first isomeric states in ${ }^{80} \mathrm{Ga}$ from the $\left(1 / 2^{+}, 5 / 2^{+}\right)$ground state of ${ }^{81} \mathrm{Zn}$. The apparent $\beta$-n feeding, $I_{\beta n}(E)$, is obtained from the intensity balance. Internal conversion is included, specifically for the $75-\mathrm{keV}$ transition, by taking coefficients from Ref. [45] and assuming dipole transitions. It is worth 


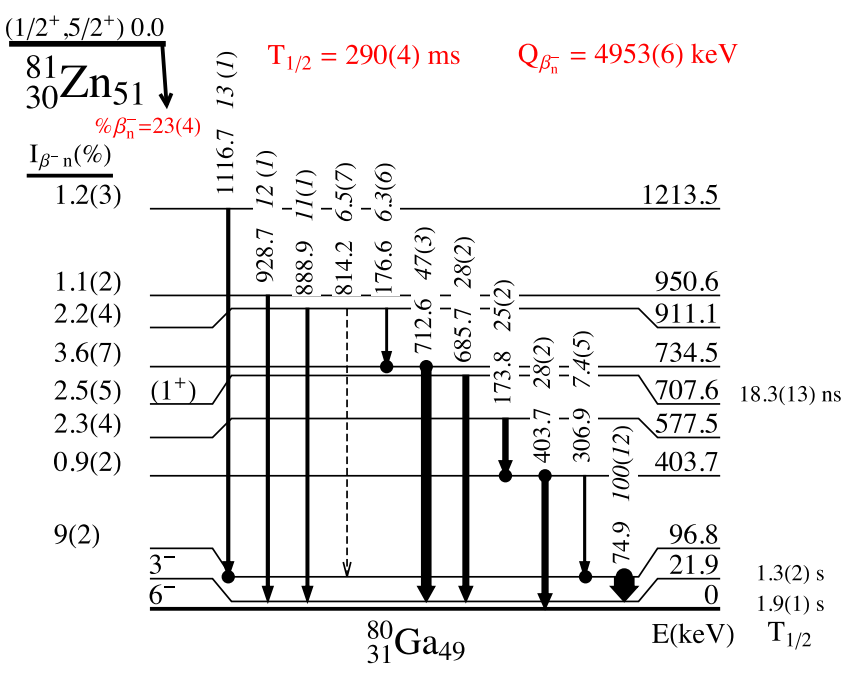

FIG. 7. Levels in ${ }^{80} \mathrm{Ga}$ populated in the $\beta^{-} \mathrm{n}$ decay of ${ }^{81} \mathrm{Zn}$ from our work. The half-lives of the $21.9-\mathrm{keV}$ and ground state were previously reported in Ref. [39].

noting that most of the population from the $\beta^{-} \mathrm{n}$ decay of ${ }^{81} \mathrm{Zn}$ (indirectly) proceeds to the $3^{-}$state at $22 \mathrm{keV}$. The second isomer, with spin parity $1^{+}$, is confirmed at $708 \mathrm{keV}$. We measured its half-life to be $T_{1 / 2}=18.3(13) \mathrm{ns}$ using triple coincidences between the $\beta$ and two HPGe detectors. Our half-life for the $708-\mathrm{keV}$ state has slightly less precision but is in perfect agreement with the value determined in Ref. [25].

\section{E. Half-lives of the excited states of ${ }^{81} \mathrm{Ga}$}

Two strong sequential transitions of 351 and $452 \mathrm{keV}$ are observed in the level scheme of ${ }^{81} \mathrm{Ga}$ (Fig. 5 and Fig. 6). The first one deexcites the first excited state of the same energy while the second one comes from the $802-\mathrm{keV}$ energy level. Selecting the $351-\mathrm{keV}$ transition in the HPGe detectors and the $452-\mathrm{keV}$ one in the $\mathrm{LaBr}_{3}(\mathrm{Ce})$ detectors,

TABLE II. Gamma transitions in ${ }^{80} \mathrm{Ga}$ populated in the $\beta^{-}$-n decay of ${ }^{81} \mathrm{Zn}$. Intensities relative to the $74.9-\mathrm{keV}$ transition, placements in the level scheme, and main $\gamma-\gamma$ coincidences are listed where available.

\begin{tabular}{lcccc}
\hline \hline$E_{\gamma}(\mathrm{keV})$ & $E_{\text {level }}^{i}(\mathrm{keV})$ & $E_{\text {level }}^{f}(\mathrm{keV})$ & $I_{\gamma}(\%)$ & $\gamma-\gamma$ \\
\hline 74.91 & 96.83 & 21.93 & 10012 & 307,1117 \\
173.84 & 577.52 & 403.72 & 252 & 404 \\
176.61 & 911.14 & 734.53 & 6.36 & 713 \\
306.92 & 403.72 & 96.83 & 7.45 & 75 \\
403.72 & 403.72 & 0.0 & 282 & 174 \\
685.71 & 707.63 & 21.93 & 282 & \\
712.66 & 734.53 & 21.93 & 473 & 177 \\
$814.2^{\mathrm{a}} 2$ & 911.14 & 96.83 & 6.57 & \\
888.93 & 911.14 & 21.93 & 111 & \\
928.75 & 950.66 & 21.93 & 121 & \\
1116.73 & 1213.54 & 96.83 & 131 & 75 \\
\hline \hline
\end{tabular}

${ }^{\text {a Weak transition, not observed in } \gamma-\gamma \text { coincidences. Tentatively }}$ placed in the level scheme. the $\beta \mathrm{LaBr}_{3}(\mathrm{Ce})$ time difference distribution is due to the lifetime of the $802-\mathrm{keV}$ level plus the contributions from the lifetimes of higher-lying levels. By reversing the gates, selecting the 452-keV line now in the HPGe and the 351-keV one in the $\mathrm{LaBr}_{3}(\mathrm{Ce})$ detectors, the observed time delayed spectrum arises from the lifetime of both the $802-\mathrm{keV}$ and the 351-keV levels, plus the contributions from higher-lying states. The difference between the centroids of both time distributions, once corrected for the different prompt positions at 351 and $452 \mathrm{keV}$ (using the FEP response curve and their Compton background contribution), yields the mean-life of the 351-keV level. Figure 8 shows two plots that illustrate the time distributions under these conditions. The time difference between their centroids shown in the figure is not yet corrected by the effect of prompt position and the Compton background response. After corrections, the centroid shift method gives the values of $\tau=92(15) \mathrm{ps}$ for the first $\mathrm{LaBr}_{3}(\mathrm{Ce})$ detector and $80(13) \mathrm{ps}$ for the second one. We take the average of both values and uncertainties, which leads to a $T_{1 / 2}=60(10) \mathrm{ps}$ half-life.

As a cross-check we have tried to deconvolute the slope in the time spectra in $\beta \gamma(\mathrm{t})$ and $\beta \gamma \gamma(\mathrm{t})$ coincidences by selecting the $351-\mathrm{keV}$ transition in the $\operatorname{LaBr}_{3}(\mathrm{Ce})$ detectors and fixing the prompt distribution to that given by the $452-\mathrm{keV}$ transition. Although the result is limited by statistics, it is consistent with a slope that yields a half-life of the order of 50 ps.

A similar procedure to that described above for the $351-\mathrm{keV}$ level is applied to measure the lifetime of the 1936-keV state, using in this case the coincident 1585- and $2358-\mathrm{keV}$ transitions. The results are at the limit of sensitivity and yield $\tau=20(18) \mathrm{ps}$ and $\tau=6(16) \mathrm{ps}$, respectively. We take the average value of $\tau=13(17)$ ps resulting in a onesigma upper limit of $T_{1 / 2} \leqslant 21 \mathrm{ps}$ for this level.

The half-life of the second excited state at $802 \mathrm{keV}$ is measured by absolute comparison using parallel transitions [35]. The high-lying states in ${ }^{81} \mathrm{Ga}$ are characterized by short half-lives below $\sim 1$ ps. Several high-energy $\gamma$ transitions are in coincidence with the 351-keV $\gamma$ ray (Fig. 5 and Fig. 6). By selecting those in the HPGe detectors and the $351-\mathrm{keV}$ one in the $\mathrm{LaBr}_{3}(\mathrm{Ce})$ detector, the $\beta$ - $\mathrm{LaBr}_{3}(\mathrm{Ce})$ time difference will arise from the $351-\mathrm{keV}$ state lifetime. This can be compared with the time distribution resulting from the selection of the $452-\mathrm{keV}$ transition in the HPGe and the $351-\mathrm{keV}$ one in the $\mathrm{LaBr}_{3}(\mathrm{Ce})$ detectors, which is due to both the 351and $802-\mathrm{keV}$ lifetimes. The difference between centroid positions, once corrected by the calibrations, gives an average of $\tau=34(22)$ ps or $T_{1 / 2}=23(16) \mathrm{ps}$ for the $802-\mathrm{keV}$ level half-life.

The previously unknown half-lives obtained from this measurement are summarized in Table III. Using the lifetimes and $\gamma$-ray branching from our level scheme, the transition probabilities for the deexciting lines have been calculated for the most probable multipolarities. The theoretical evaluation of conversion coefficients [45] for these transitions show that all of them are well below $1 \%$ and thus were neglected. Pure transitions are assumed for the experimental values. 

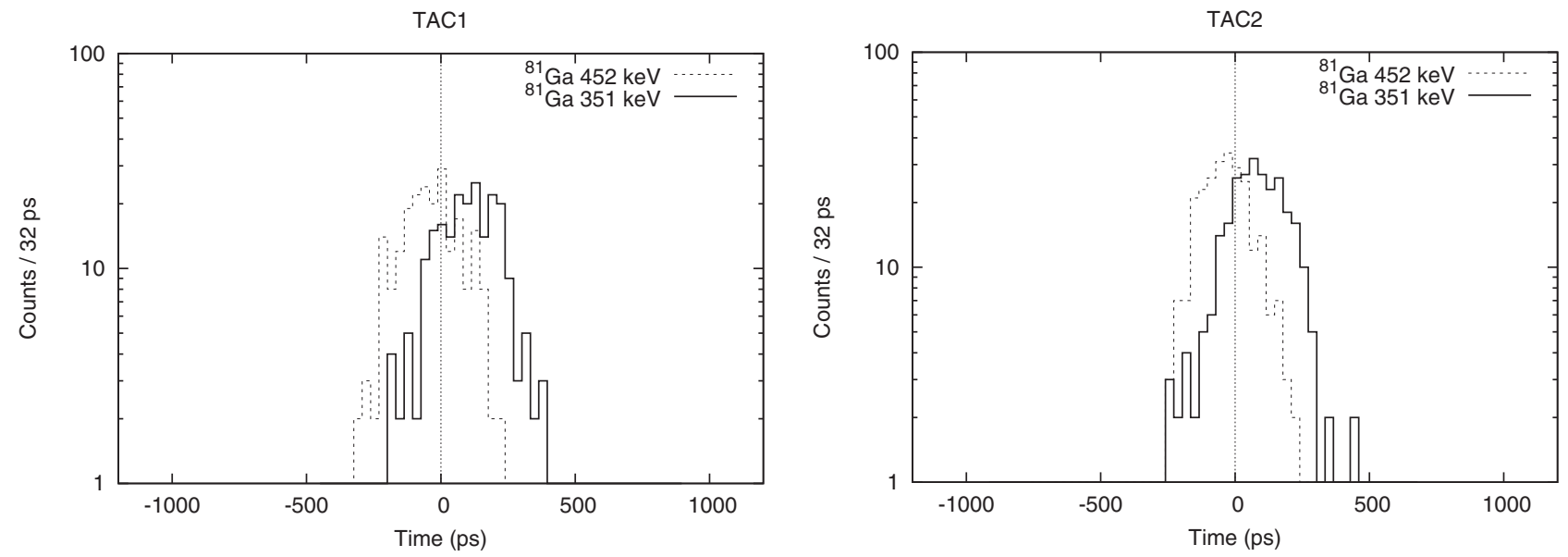

FIG. 8. Time spectra obtained in triple $\beta \gamma \gamma(\mathrm{t})$ coincidences with the 351-keV $\gamma$ transition selected in the HPGe detectors and the 452-keV

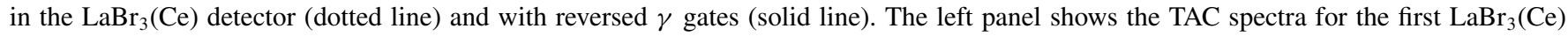
and the right panel the spectra for the second $\mathrm{LaBr}_{3}(\mathrm{Ce})$. The time distributions do not include timing corrections of the prompt positions and Compton background contributions. Once corrected for these, the difference of the centroid positions of the time distributions yields the mean-life of the 351-keV level. See text for details.

According to the measured $B(X L)$ values, both the 351- and 452-keV transitions are consistent with having a predominant $M 1$ character as in the case of the $345-\mathrm{keV}$ transition in the $N=50{ }^{85} \mathrm{Br}$ isotone [46] connecting the well-established $3 / 2^{-}$and $5 / 2^{-}$states, as shown in Fig. 9. Based on systematics, an $M 1$ multipolarity is also suggested in Ref. [21] for the $307-\mathrm{keV}$ transition connecting the tentatively assigned $\left(3 / 2^{-}\right)$first excited state and $\left(5 / 2^{-}\right)$ground state in ${ }^{83}$ As, which was also measured in Ref. [47].

\section{SHELL-MODEL CALCULATIONS}

Large-scale shell-model calculations of nuclear states of ${ }^{81} \mathrm{Ga}$ have been performed. Two state-of-the-art effective interactions were implemented into the NuShellX@MSU [54] and ANTOINE [55] codes. The first interaction, labeled JUN45, was developed by Honma et al. in 2009 [56] and it was focused in the $p f$ shell with a ${ }^{56} \mathrm{Ni}$ core and contains the $1 p_{3 / 2}, 0 f_{5 / 2}, 1 p_{1 / 2}$, and $0 g_{9 / 2}$ single-particle orbits. The interaction reproduces the experimental data of low-lying states in the $N=49$ isotones, Ge isotopes near $N=40$, and $N=Z$ nuclei with $A=64-70$, but the valence space may not contain all the degrees of freedom necessary to account for all the features of the nuclear structure of the region [56].

Another effective interaction, called jj44b, which made successful predictions for nuclei near ${ }^{78} \mathrm{Ni}$, was created in 2004 by Lisetskiy et al. [54]. It was constructed with a ${ }^{56} \mathrm{Ni}$ core for the neutron space and a ${ }^{78} \mathrm{Ni}$ core for the proton space. The Hamiltonian was also based on the Bonn-C NN potential including four single-particle energies and $65 T=1$ two-body matrix elements. The interaction was later updated [19] to better describe the structure of ${ }^{81} \mathrm{Ga}$ and it has been shown to reproduce the properties of the heavier isotopes of $\mathrm{Ga}$ [57]. Here we employ the original jj44b interaction.

The energy levels of ${ }^{81} \mathrm{Ga}$ obtained with the JUN45 and $\mathrm{jj} 44 \mathrm{~b}$ interactions are compared to our experimental results in Fig. 10. The calculations using the JUN45 interaction achieve a good agreement with the experiment for the excitation energy of the low-lying states, but tend to overestimate the energy of the negative-parity levels in the $1-$ to $2-\mathrm{MeV}$ region. On the other hand the $\mathrm{jj} 44 \mathrm{~b}$ interaction fails to reproduce the energy of the second-excited state, but achieves a better description of the level density in the $1-$ to $2-\mathrm{MeV}$ region.

TABLE III. Summary of half-lives of excited states in ${ }^{81} \mathrm{Ga}$, and experimental $B(M 1)$ and $B(E 2)$ reduced transition probabilities for the deexciting transitions, assuming pure multipolarities. They are compared to the theoretical values calculated with the JUN45 and jj44b effective interactions (see text for details).

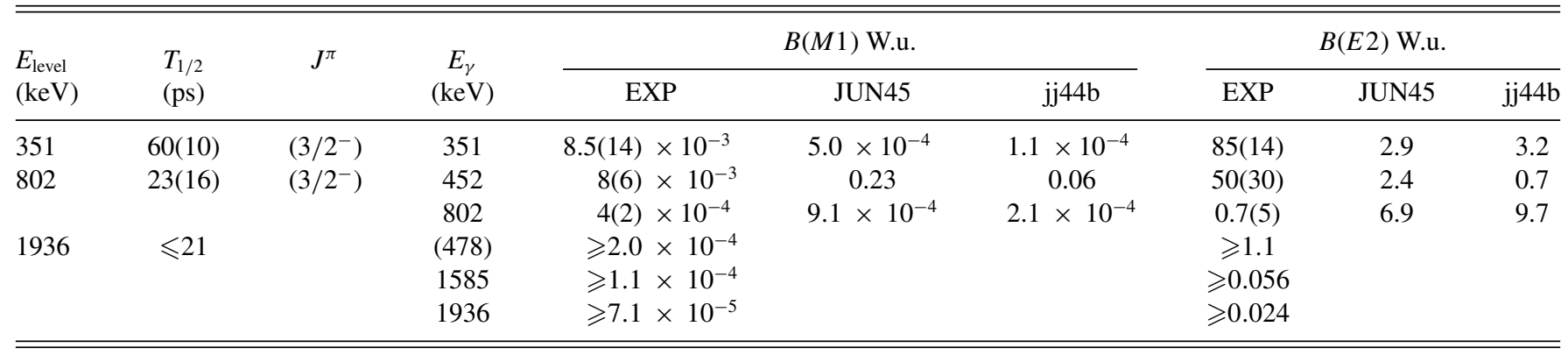




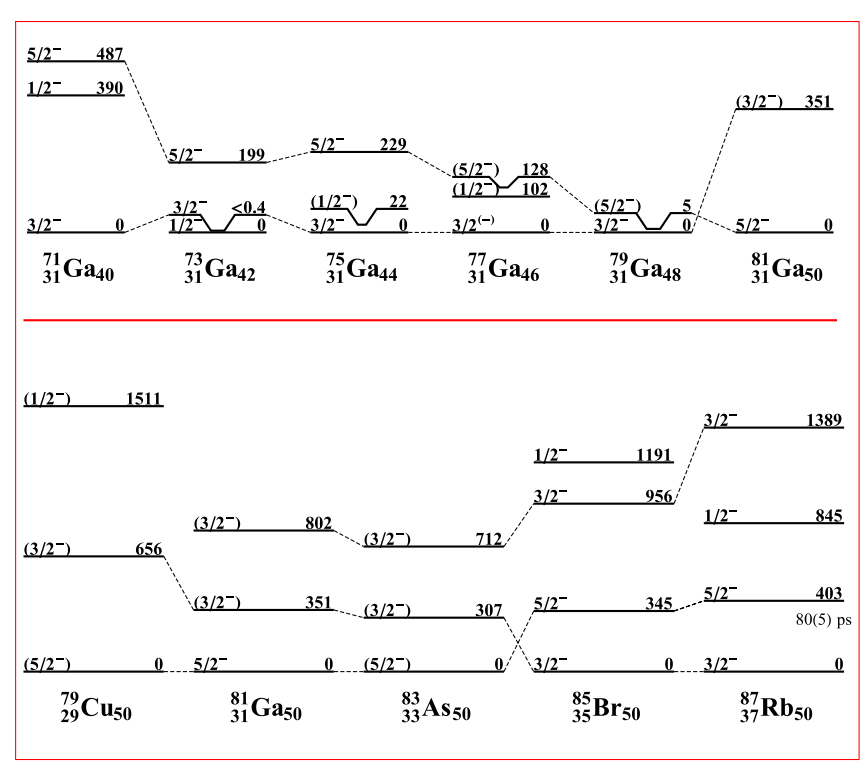

FIG. 9. Top: Level systematics of $N=40-50 \mathrm{Ga}$ isotopes. Bottom: $N=50$ isotones. The low-lying levels of $\mathrm{Ga}$ isotopes are taken from Refs. [38,48-50], except for ${ }^{81} \mathrm{Ga}$ from this work, while the structure of the $N=50$ isotones is based on Refs. [10,51-53].

Both sets of calculations give the lowest-lying positiveparity state as a spin and parity of $9 / 2^{+}$that will arise from the $\pi\left(g_{9 / 2}\right)$ configuration, at around $3 \mathrm{MeV}$. The higher-lying positive-parity states obtained from the calculations in this restricted model space must arise from the coupling of a $\pi\left(g_{9 / 2}\right)$ proton to a proton pair in the negative-parity orbitals. These states cannot be related to the experimentally observed ones, since the latter should have a neutron intruder nature (1p-1h neutron configurations) in order to be connected to the ${ }^{81} \mathrm{Zn}$ ground state via Gamow-Teller transitions.

The occupation probabilities predicted with both interactions for the lowest-lying states are summarized in Table IV. Both sets of shell-model calculations are able to properly reproduce the ground-state spin parity to be $5 / 2^{-}$, in agreement with the experimental value [20]. The proton occupation probability for the $\pi\left(f_{5 / 2}\right)^{3}$ configuration is $79 \%$ with JUN45 and $75 \%$ with jj44b. A spin parity of $3 / 2^{-}$is calculated for the first and the second excited states with both interactions, although their energies differ considerably. According to the calculations using the jj44b interaction, the 351-keV level has a single-particle $\pi\left(p_{3 / 2}\right)$ character, with a large occupation value for the $\pi\left(f_{5 / 2}\right)^{2} \otimes \pi\left(p_{3 / 2}\right)$ configuration, whereas the $802-\mathrm{keV}$ level has a preferred $\pi\left(f_{5 / 2}\right)^{3}$ configuration. The calculations for ${ }^{81} \mathrm{Ga}$ using the PFSDG-U interaction [5] reported in Ref. [22] support this description.

The calculated reduced transition probabilities for the $\gamma$ rays deexciting the lowest-lying excited states are given in Table III and compared with the measured values based on the experimental lifetimes and branching ratios. Only parityconserving transitions of the lowest multipolarities are considered due to the negative-parity nature of the low-lying states. Here effective charges of $e_{\pi}=+1.5 e$ and $e_{\nu}=+1.1 e$ were used, along with a quenched $g$ factor of $0.7 g_{s}$,free. The harmonic oscillator potential used was $41 A^{-1 / 3} \mathrm{MeV}$, as recommended in Ref. [58], which was found to better reproduce the transition rates in this region.

\section{DISCUSSION}

\section{A. Ground-state feeding of ${ }^{81} \mathbf{G a}$ and ground-state spin parity of ${ }^{81} \mathrm{Zn}$}

The structure of the nuclei immediately north of ${ }^{78} \mathrm{Ni}$ is defined by the ordering and occupation probabilities of the $g_{9 / 2}, d_{5 / 2}, s_{1 / 2}, g_{7 / 2}$, and $d_{3 / 2}$ neutron orbitals and the $f_{7 / 2}, p_{3 / 2}, p_{1 / 2}$, and $f_{5 / 2}$ proton orbitals. For the $\mathrm{Cu}$ $(Z=29)$ isotopic chain, an inversion of the ordering of the $p_{3 / 2}$ and $f_{5 / 2}$ states above ${ }^{75} \mathrm{Cu}$ has been observed as the neutron $g_{9 / 2}$ orbital is being filled. This has been interpreted as the effect of the monopole neutron-proton tensor interaction [59].

The ${ }^{81} \mathrm{Ga}$ ground-state spin parity has been established as $5 / 2^{-}$from collinear laser spectroscopy performed at ISOLDE [20]. For the ${ }^{81} \mathrm{Zn}$ ground state, positive-parity states with a single-particle character, $v s_{1 / 2}$ or $v d_{5 / 2}$, have been proposed, leading to either $1 / 2^{+}$spin-parity assignment, matching the extrapolation of the $1 / 2$ state energies in the region [19], or $5 / 2^{+}$, which is consistent with the systematics of $N=51$ isotones. $\beta$-decay transitions from ${ }^{81} \mathrm{Zn}$ to low-lying states in ${ }^{81} \mathrm{Ga}$ are expected to proceed via forbidden transitions, since the Gamow-Teller operator will populate daughter states at much higher energy. Using the systematics for forbidden decays [44] and based on the firm spin-parity assignment $5 / 2^{-}$for the ground state of ${ }^{81} \mathrm{Ga}$, two options are possible: a first-forbidden decay from the $5 / 2^{+}$to $5 / 2^{-}$states with $\log f t>5.9$ or a first-forbidden unique decay from $1 / 2^{+}$to $5 / 2^{-}$, more hindered and with $\log f t>7.5$.

In Ref. [24] Padgett et al. ruled out the previous suggestion of $1 / 2^{+}$[19] for the ground-state spin parity of ${ }^{81} \mathrm{Zn}$. Instead a $5 / 2^{+}$assignment for the ${ }^{81} \mathrm{Zn}$ ground state was proposed [24] based on the apparent $\beta$-decay feeding to the ${ }^{81} \mathrm{Ga} 5 / 2^{-}$ ground state. Our experimental data yield a $\beta$ ground-state feeding compatible with zero, with a $\log f t$ larger than 6.8. We note that high-energy transitions can still be missed in our detection set-up and that, on the contrary, some of the high-energy transitions that are unambiguously identified as belonging in ${ }^{81} \mathrm{Ga}$ have been tentatively placed in the level scheme as directly feeding the ground state based on the lack of observed coincidences. Thus the experimental value needs to be taken with caution. Nonetheless, the observed negligible direct $\beta$ feeding to the ground state is in contrast to the previous experiment [24]. This is because many weak $\gamma$ transitions depopulating high-lying states in ${ }^{81} \mathrm{Ga}$ have been added in our study, which has a dramatic effect on the ground-state $\gamma$ feeding intensity and decreases the apparent direct groundstate $\beta$ branching from the previous $52 \%$ to our $\leqslant 2.4 \%$. It is therefore very risky to base spin-parity assignments on the apparent $\beta$ feeding. From our measurement none of the possible spin-parity assignments of ${ }^{81} \mathrm{Zn}$ can be ruled out, since a first forbidden unique $1 / 2^{+}$to $5 / 2^{-}$transition, and thus a $1 / 2^{+81} \mathrm{Zn}$ ground-state spin parity, is still possible.

In any case, the role of first-forbidden transitions to lowlying negative-parity states in ${ }^{81} \mathrm{Ga}$ is not as relevant as 


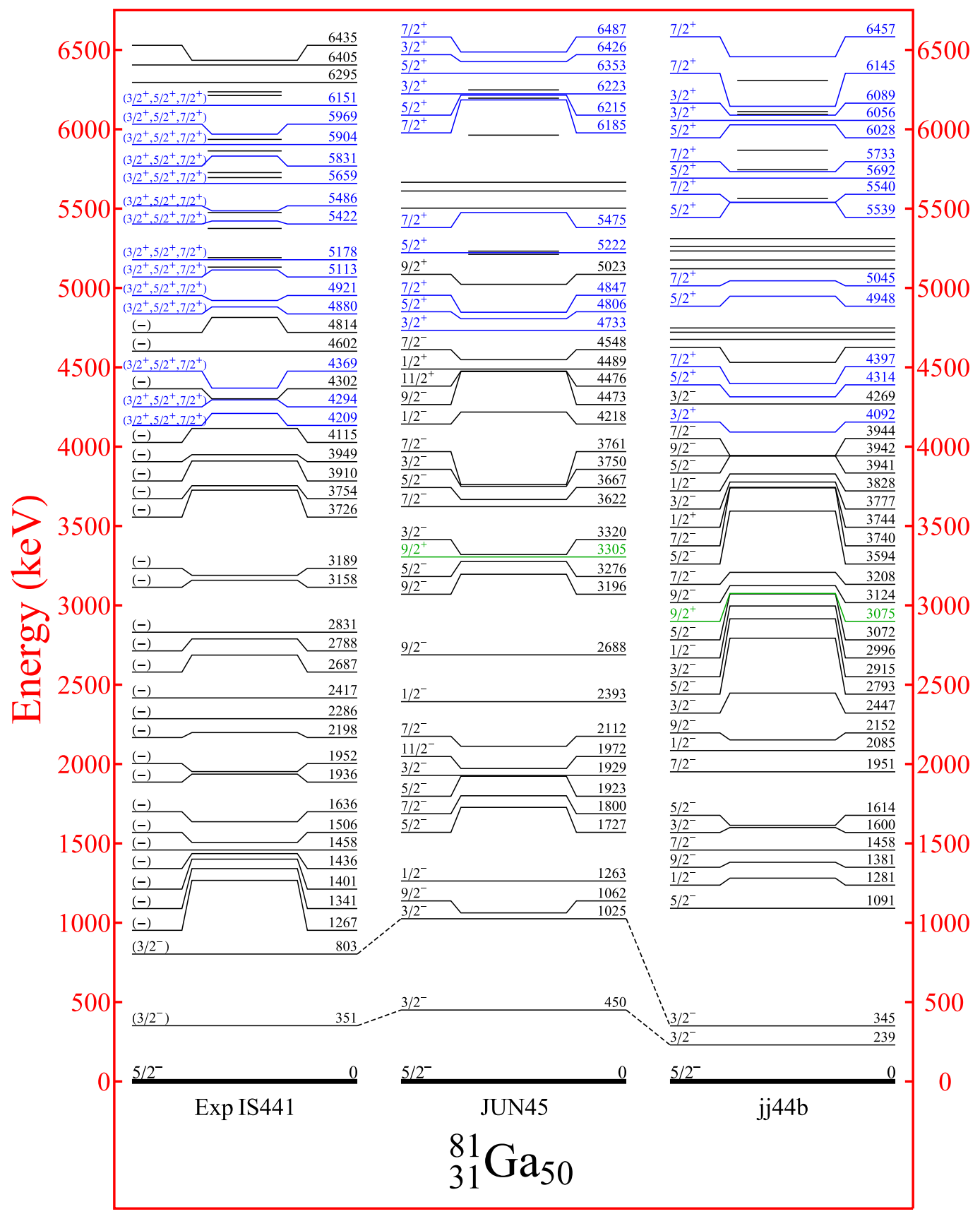

FIG. 10. Shell-model calculations with the JUN45 and jj44b interactions for ${ }^{81} \mathrm{Ga}$ compared to the experimental data measured in this work. The experimental spins were tentatively placed based on the $\beta$-feeding considerations. The positive-parity states are marked in blue, except for the calculated $9 / 2^{+}$state, which is highlighted in green.

previously proposed. A large fraction of the $\beta$-decay population may still proceed to higher-lying positive-parity states via allowed transitions. This is ratified by the sizable $\beta$-delayed neutron emission probability, which points to $\beta$ feeding via GT transitions to high-energy levels above the neutron separation energy in ${ }^{81} \mathrm{Ga}$.

\section{B. Low-lying structure of ${ }^{81} \mathbf{G a}$}

In our experiment we have measured the first-excited $351-\mathrm{keV}$ state half-life to be $T_{1 / 2}=60(10) \mathrm{ps}$. Assuming that the transition connects states of negative parity, the $B(M 1)$ rate for pure $M 1$ multipolarity is $8.5(14) \times 10^{-3}$ W.u., whereas the pure $E 2$ rate would be a very collective $85(14)$ W.u. The reduced transition probabilities thus point to a retarded $M 1$ transition which is consistent with the results of the shell-model calculations (Table III and the systematics in Ref. [60]). This suggests a $\pi p_{3 / 2}$ dominant configuration for the 351-keV level (see Table IV). Due to this fact, the 351$\mathrm{keV} \gamma$ ray would be slightly hindered due to the $l$-forbidden character of a $\pi p_{3 / 2}$ to $\pi f_{5 / 2}$ transition. 
TABLE IV. Occupation probabilities for the proton configurations obtained with the JUN45 and jj44b interactions for the first three states in ${ }^{81} \mathrm{Ga}$.

\begin{tabular}{|c|c|c|c|c|c|}
\hline \multirow{2}{*}{$\begin{array}{l}E_{\text {level }} \\
(\mathrm{keV})\end{array}$} & \multirow{2}{*}{$J^{\pi}$} & \multicolumn{2}{|c|}{ JUN45 } & \multicolumn{2}{|c|}{$\mathrm{jj} 44 \mathrm{~b}$} \\
\hline & & $\pi\left(f_{5 / 2}\right)^{3}$ & $\pi\left(f_{5 / 2}\right)^{2} p_{3 / 2}$ & $\pi\left(f_{5 / 2}\right)^{3}$ & $\pi\left(f_{5 / 2}\right)^{2} p_{3 / 2}$ \\
\hline 0 & $5 / 2^{-}$ & $79 \%$ & $4 \%$ & $75 \%$ & $2 \%$ \\
\hline 351 & $\left(3 / 2^{-}\right)$ & $38 \%$ & $51 \%$ & $5 \%$ & $78 \%$ \\
\hline 802 & $\left(3 / 2^{-}\right)$ & $48 \%$ & $41 \%$ & $76 \%$ & $5 \%$ \\
\hline
\end{tabular}

A very similar structure is found in odd $N=50$ nuclei with $Z>28$, as shown in Fig. 9, in particular for the neighboring isotone ${ }^{83} \mathrm{As}$. For the $Z=37{ }^{87} \mathrm{Rb}$ isotone, the $3 / 2^{-}$and $5 / 2^{-}$ levels are already reversed, the ground state having a spin of $3 / 2^{-}$. The $403-\mathrm{keV} 5 / 2^{-}$level has a very similar half-life of $T_{1 / 2}=80(5)$ ps [61] to the $351-\mathrm{keV}$ one in ${ }^{81} \mathrm{Ga}$, which gives a $B(M 1)$ to within a factor of 2 for the $403-\mathrm{keV}$ transition to the ground state compared to the $351-\mathrm{keV}$ transition in ${ }^{81} \mathrm{Ga}$. The dominant $\pi p_{3 / 2}$ single-particle configuration of the $351-\mathrm{keV}$ level is in accord with a narrow proton gap of the order of $500 \mathrm{keV}$ between the $f_{5 / 2}$ and $p_{3 / 2}$ orbitals, as predicted for ${ }^{79} \mathrm{Cu}$ by shell-model calculations $[58,62]$, but at odds with what was claimed in Ref. [63].

For the second excited state at $802 \mathrm{keV}$, the calculations, especially those with the JUN45 interaction (which achieve a better agreement with the experimental excitation energies) show a strong admixture of the $\pi\left(f_{5 / 2}\right)^{3}$ cluster configuration and the $\pi\left(f_{5 / 2}\right)^{2} p_{3 / 2}$ one. This gives rise to a $3 / 2^{-}$spin parity. Our measured half-life for this level is consistent with a 3/2- assignment, and an $M 1452-\mathrm{keV}$ deexciting transition, whereas for the $802-\mathrm{keV}$ transition the experimental result allows for a possible $E 2$ component.

Both of the 351- and 802-keV levels are fed from positiveparity high-energy levels. These may be characterized by the occupation of $\pi g_{7 / 2}, \pi d_{5 / 2}$, and $\pi d_{3 / 2}$ proton single-particle states, or by the coupling of proton orbitals to neutron particlehole states (thus requiring breaking of neutron pairs across the $N=50$ gap). The deexcitations from these high-lying states, which likely have spins between $1 / 2^{+}$and $7 / 2^{+}$to $3 / 2^{-} 351-$ and $802-\mathrm{keV}$ states take place via $E 1$ high-energy transitions with energies higher than $3.8 \mathrm{MeV}$. In view of the $3.5-\mathrm{MeV}$ $N=50$ energy gap measured for ${ }^{81} \mathrm{Ga}$ by Hakala et al. [64] the $3859-\mathrm{keV}$ transition which connects the $\beta$-fed $4209-\mathrm{keV}$ level to the $351-\mathrm{keV}$ state gives a rough estimate of the $N=50$ energy gap from our data.

Nine other excited states are experimentally found in ${ }^{81} \mathrm{Ga}$ below $2 \mathrm{MeV}$. The calculations reproduce the level density of these negative-parity states. In a simplistic model where a quasiparticle is coupled to the ${ }^{80} \mathrm{Zn}$ core, four states arising from the coupling of the $\pi\left(p_{3 / 2}\right)$ orbit to the $2^{+}$core in ${ }^{80} \mathrm{Zn}$, would have spins of $1 / 2^{-}, 3 / 2^{-}, 5 / 2^{-}$, and $7 / 2^{-}$, whereas the $\pi\left(f_{5 / 2}\right)$ coupling to the $2^{+}$level will give rise to the five states with spins ranging from $1 / 2^{-}$to $9 / 2^{-}$. An alternative description based on a $\pi\left(f_{5 / 2}\right)^{3}$ cluster configuration provides a similar picture. The $\pi\left(f_{5 / 2}\right)^{3}$ configuration yields $3 / 2^{-}$, $5 / 2^{-}$, and $9 / 2^{-}$spins, with the $9 / 2^{-}$found at higher energies, and their couplings to the $2^{+}$of ${ }^{78} \mathrm{Ni}$ in this case will provide the observed levels. It is worth noting that both theoretical calculations reproduce rather well the excitation energy of the $9 / 2^{-}$level at $1341.0 \mathrm{keV}$ belonging to the $\pi\left(f_{5 / 2}\right)^{3}$ configuration. This is consistent with the calculations presented in Ref. [22].

A high density of levels in the region from 1 to $2 \mathrm{MeV}$ can be observed as well in the level scheme of ${ }^{83} \mathrm{As}$ [51], with striking similarity to that of ${ }^{81} \mathrm{Ga}$. The level scheme of ${ }^{85} \mathrm{Br}$ [46], populated by the $\beta$ decay of ${ }^{85} \mathrm{Se}$ has a very similar structure too. Of these levels in ${ }^{81} \mathrm{Ga}$, the $1936-\mathrm{keV}$ state is strongly populated from the higher-lying $4295-\mathrm{keV}$ positiveparity state. We have measured a 21-ps upper half-life limit for the former, which does not allow us to unambiguously identify the multipolarity of the depopulating 1936- and $1585-\mathrm{keV}$ transitions. However, the decay pattern to the ground and first-excited levels, and the direct feeding from positive-parity states, makes a spin-parity assignment of $3 / 2^{-}$or $5 / 2^{-}$likely for this state.

\section{Positive-parity states}

As mentioned above, the lowest positive-parity state is the $9 / 2^{+}$one predicted at energies close to 3.0 and $3.3 \mathrm{MeV}$, depending on the interaction. This state has an expected main $\pi g_{9 / 2}$ configuration and would not be directly populated by the ${ }^{81} \mathrm{Zn} \beta$ decay from a $1 / 2^{+}$ground state, and would have a limited feeding from a $5 / 2^{+}$ground state, yielding a high $\log f t$ value. Although the single-particle $v g_{7 / 2}$ orbit is shown to be at higher energy [21], any admixture of a $v g_{7 / 2}$ component in the ${ }^{81} \mathrm{Zn}$ ground-state wave function would lead to an enhancement of allowed Gamow-Teller (GT) $\beta$ transtions to the $\pi g_{9 / 2}$ orbit. In any case, indirect population of the $9 / 2^{+}$state in ${ }^{81} \mathrm{Ga}$ should be possible. The systematics near $A=81$ suggests a long half-life for this state due to the $M 2$ behavior of the $\gamma$ transition which would connect it to the $5 / 2^{-}$ground state. No such long lifetime, nor decay to lower energy $7 / 2^{-}$or $9 / 2^{-}$levels, could be observed in our measurement.

The allowed GT $\beta$ decay from the ${ }^{81} \mathrm{Zn}$ ground-state neutron $v\left(d_{5 / 2}\right)$ or $v\left(s_{1 / 2}\right)$ configuration populates high-energy states in the ${ }^{81} \mathrm{Ga}$ daughter, since there are no low-lying positive-parity states available. The positive states would have to originate from the coupling of the odd proton orbitals $\left(p_{3 / 2}, f_{5 / 2}\right.$, and $\left.p_{1 / 2}\right)$ to neutron particle-hole states, therefore implying the breaking of a neutron pair inside the $N=50$ shell. These cross-shell states arising from the excitation of the ${ }^{78} \mathrm{Ni}$ core give an idea of the magnitude of the $N=50$ shell gap, as discussed by Winger et al. [51] in the $\beta$ decay of ${ }^{83} \mathrm{Ge}$ to ${ }^{83} \mathrm{As}$, and Padgett and coworkers for ${ }^{81} \mathrm{Ga}$ [24].

The GT $\beta$ decays to these core-excited states must arise from the decay of neutrons in ${ }^{81} \mathrm{Zn}$ in the $f$ and $p$ orbitals, which are strongly bound. Due to the reduced energy window the $\beta$ feeding would be reduced, but, in spite of the Fermi factor, these GT decays may still be favoured compared to the first-forbidden decays to low-lying negative-parity states. The large $\mathrm{P}_{n}$ value measured for ${ }^{81} \mathrm{Zn}$ suggests a significant role of such allowed $\beta$ transitions to high-lying states above the neutron separation energy in ${ }^{81} \mathrm{Ga}$. Several levels with low apparent $\log f t$ values can be identified in the $4-$ to $5-\mathrm{MeV}$ 
energy range. In our work we observe strong direct population to the levels at 4209, 4295, 4369, 4880, 4921, 5178, and 5422 $\mathrm{keV}$, and to some others at higher energies. These states are not included in our shell-model calculations due to the restricted model spaces. Assuming a $1 / 2^{+}$or $5 / 2^{+}$ground state for ${ }^{81} \mathrm{Zn}$, positive-parity assignments for these levels with $1 / 2$, $3 / 2,5 / 2$, and $7 / 2$ spin values can be made. An identical situation can be observed in the $N=50$ isotones ${ }^{83} \mathrm{As}$ [51] and ${ }^{85} \mathrm{Br}$ [46], populated following the $\beta$ decay of ${ }^{83} \mathrm{Ge}$ and ${ }^{85} \mathrm{Se}$, respectively.

\section{SUMMARY AND CONCLUSIONS}

The high purity and intensity of the $\mathrm{Zn}$ beams delivered by the ISOLDE facility at CERN have made it possible to obtain about 10-fold higher statistics than previous studies [24]. The level scheme of the semimagic $N=50$ nucleus ${ }^{81} \mathrm{Ga}$ has been significantly expanded with 47 new levels and $70 \gamma$ transitions in the energy range up to $6.5 \mathrm{MeV}$. Most of these levels are very close to the neutron separation energy. The 290(4)-ms half-life of ${ }^{81} \mathrm{Zn}$ measured in this work is in good agreement with the literature [4,24].

The direct $\beta$ feeding to the ${ }^{81}$ Ga ground state measured in our experiment is negligible within the error bars, and much lower than proposed previously; it is thus compatible with both $5 / 2^{+}$and $1 / 2^{+}$assignments for the ${ }^{81} \mathrm{Zn}$ ground state. We could not identify the $9 / 2^{+}$state seen in other $N=50$ isotones and also predicted by our shell-model calculations to lie at around $3 \mathrm{MeV}$. We have measured a $\beta$-delayed neutron emission probability value of $23(4) \%$ for the decay of ${ }^{81} \mathrm{Zn}$. This is more precise but also consistent with $30(13) \%$ measured by Hosmer et al. [3], but two- $\sigma$ away from the recent value reported by Padgett and coworkers of $12(4) \%$ [24].

The level scheme of ${ }^{80} \mathrm{Ga}$ populated following the $\beta$-delayed neutron emission from ${ }^{81} \mathrm{Zn}$ was constructed for the first time and it is in agreement with that described in Ref. [25] from the $\beta$ decay of ${ }^{80} \mathrm{Ga}$, including the low-lying $22-\mathrm{keV}$ isomer. Our measurements also confirm the existence of the 708-keV isomer with an 18.3(13)-ns half-life.

We have measured the half-life of the first excited state in ${ }^{81} \mathrm{Ga}$ to be $T_{1 / 2}=60(10) \mathrm{ps}$, which indicates an $l$-forbidden $M 1$ transition of $351 \mathrm{keV}$ to the $5 / 2^{-}$ground state. This in turn points to a transition between states with main $\pi p_{3 / 2}$ and $\pi f_{5 / 2}$ configurations. This is supported by both the
$N=50$ systematics and by our shell-model calculations, where the dominant occupations for the ground and firstexcited states are found, and in agreement with earlier findings [19]. The calculated occupation probability and our experimental results suggest a main $\pi\left(f_{5 / 2}\right)^{2} \otimes \pi\left(p_{3 / 2}\right)^{1}$ configuration for the first excited state of ${ }^{81} \mathrm{Ga}$. The calculated transition rate supports this assignment, too. For the second excited state a half-life of 23(16) ps is measured. This value provides $B(M 1)=8(6) \times 10^{-3}$ W.u. and $B(E 2)=51(35)$ W.u. (Table III) reduced probabilities which, together with the shell-model results, allows us to propose a $\pi\left(f_{5 / 2}\right)^{3}$ cluster configuration and a $3 / 2^{-}$spin-parity assignment for this state.

A high density of negative-parity levels can be observed in the region from 1 to $2 \mathrm{MeV}$ of the level scheme of ${ }^{81} \mathrm{Ga}$. This is consistent with $\pi\left(p_{3 / 2}\right)$ and $\pi\left(f_{5 / 2}\right)$ single-particle states coupled to the $2^{+}$core in ${ }^{80} \mathrm{Zn}$, and it is well reproduced by the shell-model calculations. These states will be of negative parity and should be populated by first-forbidden transitions if they are directly $\beta$ fed. The level scheme of the $N=50$ isotone ${ }^{83} \mathrm{As}$ [51] also shows a density of levels around 1400 $\mathrm{keV}$ much like that of ${ }^{81} \mathrm{Ga}$. A similar structure is found in the $N=50{ }^{85} \mathrm{Br}$ isotone populated by the $\beta$ decay of ${ }^{85} \mathrm{Se}$ [46]. The situation changes beyond $5 \mathrm{MeV}$ where we observe several states with sizable apparent $\beta$ feeding, which should arise from allowed transitions from the ${ }^{81} \mathrm{Zn}$ positive-parity ground state. They can be interpreted as neutron particle-hole excitations from the ${ }^{78} \mathrm{Ni}$ core.

\section{ACKNOWLEDGMENTS}

This work was supported by the Spanish MINECO through the FPA2015-65035-P and RTI2018-098868-B-I00 projects, by the U.S. DoE Grant No. DE-FG02-94ER40834, by the German BMBF Grant 05P19PKFNA, and Grupo de Física Nuclear (GFN) at UCM. The support by the European Union Seventh Framework through ENSAR (Contract No. 262010) and ISOLDE (CERN) Collaboration is acknowledged. V.P. acknowledges support by the Spanish FPI-BES-2011-045931 grant. Figures 5, 6, and 7 were created using the LevelScheme scientific figure preparation system [65]. Fast-timing electronics were provided by the Fast Timing Collaboration and MASTICON.

H.M. pioneered the $\beta \gamma \gamma(\mathrm{t})$ fast-timing method and its application to exotic nuclei around ${ }^{78} \mathrm{Ni}$ at ISOLDE; he prematurely passed away before the completion of this article.
[1] T. Otsuka, T. Suzuki, M. Honma, Y. Utsuno, N. Tsunoda, K. Tsukiyama, and M. Hjorth-Jensen, Phys. Rev. Lett. 104, 012501 (2010).

[2] C. Engelmann, F. Ameil, P. Armbruster, M. Bernas, S. Czajkowski, P. Dessagne, C. Donzaud, H. Geissel, A. Heinz, Z. Janas, C. Kozhuharov, C. Miehé, G. Münzenberg, M. Pfützner, C. Röhl, W. Schwab, C. Stéphan, K. Sümmerer, L. Tassan-Got, and B. Voss, Z. Phys. A 352, 351 (1995).

[3] P. Hosmer, H. Schatz, A. Aprahamian, O. Arndt, R. R. C. Clement, A. Estrade, K. Farouqi, K.-L. Kratz, S. N. Liddick,
A. F. Lisetskiy, P. F. Mantica, P. Möller, W. F. Mueller, F. Montes, A. C. Morton, M. Ouellette, E. Pellegrini, J. Pereira, B. Pfeiffer, P. Reeder, P. Santi, M. Steiner, A. Stolz, B. E. Tomlin, W. B. Walters, and A. Wöhr, Phys. Rev. C 82, 025806 (2010)

[4] Z. Y. Xu, S. Nishimura, G. Lorusso, F. Browne, P. Doornenbal, G. Gey, H.-S. Jung, Z. Li, M. Niikura, P.-A. Söderström, T. Sumikama, J. Taprogge, Z. Vajta, H. Watanabe, J. Wu, A. Yagi, K. Yoshinaga, H. Baba, S. Franchoo, T. Isobe, P. R. John, I. Kojouharov, S. Kubono, N. Kurz, I. Matea, K. Matsui, 
D. Mengoni, P. Morfouace, D. R. Napoli, F. Naqvi, H. Nishibata, A. Odahara, E. Şahin, H. Sakurai, H. Schaffner, I. G. Stefan, D. Suzuki, R. Taniuchi, and V. Werner, Phys. Rev. Lett. 113, 032505 (2014).

[5] F. Nowacki, A. Poves, E. Caurier, and B. Bounthong, Phys. Rev. Lett. 117, 272501 (2016).

[6] G. Hagen, G. R. Jansen, and T. Papenbrock, Phys. Rev. Lett. 117, 172501 (2016).

[7] R. Taniuchi, C. Santamaria, P. Doornenbal, A. Obertelli, K. Yoneda, G. Authelet, H. Baba, D. Calvet, F. Château, A. Corsi, A. Delbart, J. M. Gheller, A. Gillibert, J. D. Holt, T. Isobe, V. Lapoux, M. Matsushita, J. Menéndez, S. Momiyama, T. Motobayashi, M. Niikura, F. Nowacki, K. Ogata, H. Otsu, T. Otsuka, C. Péron, S. Péru, A. Peyaud, E. C. Pollacco, A. Poves, J. Y. Roussé, H. Sakurai, A. Schwenk, Y. Shiga, J. Simonis, S. R. Stroberg, S. Takeuchi, Y. Tsunoda, T. Uesaka, H. Wang, F. Browne, L. X. Chung, Z. Dombradi, S. Franchoo, F. Giacoppo, A. Gottardo, K. Hadyńska-Klęk, Z. Korkulu, S. Koyama, Y. Kubota, J. Lee, M. Lettmann, C. Louchart, R. Lozeva, K. Matsui, T. Miyazaki, S. Nishimura, L. Olivier, S. Ota, Z. Patel, E. Şahin, C. Shand, P. A. Söderström, I. Stefan, D. Steppenbeck, T. Sumikama, D. Suzuki, Z. Vajta, V. Werner, J. Wu, and Z. Y. Xu, Nature 569, 53 (2019).

[8] K. T. Flanagan, P. Vingerhoets, M. Avgoulea, J. Billowes, M. L. Bissell, K. Blaum, B. Cheal, M. De Rydt, V. N. Fedosseev, D. H. Forest, C. Geppert, U. Köster, M. Kowalska, J. Krämer, K. L. Kratz, A. Krieger, E. Mané, B. A. Marsh, T. Materna, L. Mathieu, P. L. Molkanov, R. Neugart, G. Neyens, W. Nörtershäuser, M. D. Seliverstov, O. Serot, M. Schug, M. A. Sjoedin, J. R. Stone, N. J. Stone, H. H. Stroke, G. Tungate, D. T. Yordanov, and Y. M. Volkov, Phys. Rev. Lett. 103, 142501 (2009).

[9] Y. Ichikawa, H. Nishibata, Y. Tsunoda, A. Takamine, K. Imamura, T. Fujita, T. Sato, S. Momiyama, Y. Shimizu, D. S. Ahn, K. Asahi, H. Baba, D. L. Balabanski, F. Boulay, J. M. Daugas, T. Egami, N. Fukuda, C. Funayama, T. Furukawa, G. Georgiev, A. Gladkov, N. Inabe, Y. Ishibashi, T. Kawaguchi, T. Kawamura, Y. Kobayashi, S. Kojima, A. Kusoglu, I. Mukul, M. Niikura, T. Nishizaka, A. Odahara, Y. Ohtomo, T. Otsuka, D. Ralet, G. S. Simpson, T. Sumikama, H. Suzuki, H. Takeda, L. C. Tao, Y. Togano, D. Tominaga, H. Ueno, H. Yamazaki, and X. F. Yang, Nat. Phys. 15, 321 (2019).

[10] L. Olivier, S. Franchoo, M. Niikura, Z. Vajta, D. Sohler, P. Doornenbal, A. Obertelli, Y. Tsunoda, T. Otsuka, G. Authelet, H. Baba, D. Calvet, F. Château, A. Corsi, A. Delbart, J.-M. Gheller, A. Gillibert, T. Isobe, V. Lapoux, M. Matsushita, S. Momiyama, T. Motobayashi, H. Otsu, C. Péron, A. Peyaud, E. C. Pollacco, J.-Y. Roussé, H. Sakurai, C. Santamaria, M. Sasano, Y. Shiga, S. Takeuchi, R. Taniuchi, T. Uesaka, H. Wang, K. Yoneda, F. Browne, L. X. Chung, Z. Dombradi, F. Flavigny, F. Giacoppo, A. Gottardo, K. Hadyńska-Klęk, Z. Korkulu, S. Koyama, Y. Kubota, J. Lee, M. Lettmann, C. Louchart, R. Lozeva, K. Matsui, T. Miyazaki, S. Nishimura, K. Ogata, S. Ota, Z. Patel, E. Sahin, C. Shand, P.-A. Söderström, I. Stefan, D. Steppenbeck, T. Sumikama, D. Suzuki, V. Werner, J. Wu, and Z. Xu, Phys. Rev. Lett. 119, 192501 (2017).

[11] A. Welker, N. A. S. Althubiti, D. Atanasov, K. Blaum, T. E. Cocolios, F. Herfurth, S. Kreim, D. Lunney, V. Manea, M. Mougeot, D. Neidherr, F. Nowacki, A. Poves, M. Rosenbusch, L. Schweikhard, F. Wienholtz, R. N. Wolf, and K. Zuber, Phys. Rev. Lett. 119, 192502 (2017).
[12] Y. Shiga, K. Yoneda, D. Steppenbeck, N. Aoi, P. Doornenbal, J. Lee, H. Liu, M. Matsushita, S. Takeuchi, H. Wang, H. Baba, P. Bednarczyk, Z. Dombradi, Z. Fulop, S. Go, T. Hashimoto, M. Honma, E. Ideguchi, K. Ieki, K. Kobayashi, Y. Kondo, R. Minakata, T. Motobayashi, D. Nishimura, T. Otsuka, H. Otsu, H. Sakurai, N. Shimizu, D. Sohler, Y. Sun, A. Tamii, R. Tanaka, Z. Tian, Y. Tsunoda, Z. Vajta, T. Yamamoto, X. Yang, Z. Yang, Y. Ye, R. Yokoyama, and J. Zenihiro, Phys. Rev. C 93, 024320 (2016).

[13] H. Mach, R. Gill, and M. Moszyński, Nucl. Instrum. Methods A 280, 49 (1989).

[14] M. Moszyński and H. Mach, Nucl. Instrum. Methods A 277, 407 (1989).

[15] K.-L. Kratz, H. Gabelmann, P. Möller, B. Pfeiffer, H. Ravn, and A. Wöhr, Z. Phys. A 340, 419 (1991).

[16] D. Verney, F. Ibrahim, O. Perru, O. Bajeat, C. Bourgeois, F. Clapier, E. Cottereau, C. Donzaud, S. Du, M. Ducourtieux, S. Essabaa, S. Galès, L. Gaudefroy, D. Guillemaud-Mueller, F. Hammache, F. Hosni, C. Lau, H. Lefort, F. Le Blanc, A. Mueller, N. Pauwels, J. Potier, F. Pougheon, J. Proust, B. Roussière, J. Sauvage, and O. Sorlin, Braz. J. Phys. 34, 979 (2004).

[17] U. Köster, T. Behrens, C. Clausen, P. Delahaye, V. N. Fedoseyev, L. M. Fraile, R. Gernhäuser, T. J. Giles, A. Ionan, T. Kröll, H. Mach, B. Marsh, M. Seliverstov, T. Sieber, E. Siesling, E. Tengborn, F. Wenander, and J. Van de Walle, AIP Conf. Proc. 798, 315 (2005).

[18] I. N. Borzov, Phys. Rev. C 71, 065801 (2005).

[19] D. Verney, F. Ibrahim, C. Bourgeois, S. Essabaa, S. Galès, L. Gaudefroy, D. Guillemaud-Mueller, F. Hammache, C. Lau, F. Le Blanc, A. C. Mueller, O. Perru, F. Pougheon, B. Roussière, J. Sauvage, and O. Sorlin (PARRNe Collaboration), Phys. Rev. C 76, 054312 (2007).

[20] B. Cheal, E. Mané, J. Billowes, M. L. Bissell, K. Blaum, B. A. Brown, F. C. Charlwood, K. T. Flanagan, D. H. Forest, C. Geppert, M. Honma, A. Jokinen, M. Kowalska, A. Krieger, J. Krämer, I. D. Moore, R. Neugart, G. Neyens, W. Nörtershäuser, M. Schug, H. H. Stroke, P. Vingerhoets, D. T. Yordanov, and M. Žáková, Phys. Rev. Lett. 104, 252502 (2010).

[21] E. Sahin, G. de Angelis, G. Duchene, T. Faul, A. Gadea, A. Lisetskiy, D. Ackermann, A. Algora, S. Aydin, F. Azaiez, D. Bazzacco, G. Benzoni, M. Bostan, T. Byrski, I. Celikovic, R. Chapman, L. Corradi, S. Courtin, D. Curien, U. D. Pramanik, F. Didierjean, O. Dorvaux, M. Erduran, S. Erturk, E. Farnea, E. Fioretto, G. de France, S. Franchoo, B. Gall, A. Gottardo, B. Guiot, F. Haas, F. Ibrahim, E. Ince, A. Khouaja, A. Kusoglu, G. L. Rana, M. Labiche, D. Lebhertz, S. Lenzi, S. Leoni, S. Lunardi, P. Mason, D. Mengoni, C. Michelagnoli, V. Modamio, G. Montagnoli, D. Montanari, R. Moro, B. Mouginot, D. Napoli, D. O’Donnell, J. Oliveira, J. Ollier, R. Orlandi, G. Pollarolo, F. Recchia, J. Robin, M.-D. Salsac, F. Scarlassara, R. Singh, R. Silvestri, J. Smith, I. Stefan, A. Stefanini, K. Subotic, S. Szilner, D. Tonev, D. Torres, M. Trotta, P. Ujic, C. Ur, J. Valiente-Dobón, D. Verney, M. Yalcinkaya, P. Wady, K. Wiedemann, and K. Zuber, Nucl. Phys. A 893, 1 (2012).

[22] J. Dudouet, A. Lemasson, G. Maquart, F. Nowacki, D. Verney, M. Rejmund, G. Duchêne, O. Stezowski, E. Clément, C. Michelagnoli, A. Korichi, C. Andreoiu, A. Astier, G. de Angelis, G. de France, C. Delafosse, I. Deloncle, F. Didierjean, Z. Dombradi, C. Ducoin, A. Gadea, A. Gottardo, D. Guinet, B. Jacquot, P. Jones, T. Konstantinopoulos, I. Kuti, F. Le Blanc, 
S. M. Lenzi, G. Li, R. Lozeva, B. Million, D. R. Napoli, A. Navin, R. M. Pérez-Vidal, C. M. Petrache, D. Ralet, M. Ramdhane, N. Redon, C. Schmitt, and D. Sohler, Phys. Rev. C 100, 011301(R) (2019).

[23] L. Olivier, Nuclear structure in the vicinity of ${ }^{78} \mathrm{Ni}$ : In-beam $\gamma$ ray spectroscopy of ${ }^{79} \mathrm{Cu}$ through proton knockout, $\mathrm{Ph}$.D. thesis, Université Paris-Saclay, France, 2017.

[24] S. Padgett, M. Madurga, R. Grzywacz, I. G. Darby, S. N. Liddick, S. V. Paulauskas, L. Cartegni, C. R. Bingham, C. J. Gross, K. Rykaczewski, D. Shapira, D. W. Stracener, A. J. Mendez, J. A. Winger, S. V. Ilyushkin, A. Korgul, W. Królas, E. Zganjar, C. Mazzocchi, S. Liu, J. H. Hamilton, J. C. Batchelder, and M. M. Rajabali, Phys. Rev. C 82, 064314 (2010).

[25] R. Lică, N. Mărginean, D. G. Ghiţă, H. Mach, L. M. Fraile, G. S. Simpson, A. Aprahamian, C. Bernards, J. A. Briz, B. Bucher, C. J. Chiara, Z. Dlouhý, I. Gheorghe, P. Hoff, J. Jolie, U. Köster, W. Kurcewicz, R. Mărginean, B. Olaizola, V. Paziy, J. M. Régis, M. Rudigier, T. Sava, M. Stănoiu, L. Stroe, and W. B. Walters, Phys. Rev. C 90, 014320 (2014).

[26] V. Paziy, Ultra-fast timing study of exotic nuclei around ${ }^{78} \mathrm{Ni}$ : The $\beta$ decay chain of ${ }^{81} \mathrm{Zn}$, Ph.D. thesis, Universidad Complutense de Madrid, Spain, 2016.

[27] V. Vedia, V. Paziy, L. M. Fraile, H. Mach, W. B. Walters, A. Aprahamian, C. Bernards, J. A. Briz, B. Bucher, C. J. Chiara, Z. Dlouhý, I. Gheorghe, D. Ghiţă, P. Hoff, J. Jolie, U. Köster, W. Kurcewicz, R. Lică, N. Mărginean, R. Mărginean, B. Olaizola, J.-M. Régis, M. Rudigier, T. Sava, G. S. Simpson, M. Stănoiu, and L. Stroe, Phys. Rev. C 96, 034311 (2017).

[28] U. Köster, O. Arndt, E. Bouquerel, V. Fedoseyev, H. Frånberg, A. Joinet, C. Jost, I. Kerkines, and R. Kirchner, Nucl. Instrum. Methods B 266, 4229 (2008).

[29] U. Köster, Eur. Phys. J. A 15, 255 (2002).

[30] E. Bouquerel, R. Catherall, M. Eller, J. Lettry, S. Marzari, T. Stora, and ISOLDE Collaboration, Eur. Phys. J.: Spec. Top. 150, 277 (2007).

[31] V. Fedosseev, L.-E. Berg, N. Lebas, O. Launila, M. Lindroos, R. Losito, B. Marsh, F. Österdahl, T. Pauchard, G. Tranströmer, and J. Vannesjö, Nucl. Instrum. Methods B 266, 4378 (2008).

[32] V. Vedia, M. Carmona-Gallardo, L. M. Fraile, H. Mach, and J. M. Udías, Nucl. Instrum. Methods A 857, 98 (2017).

[33] V. Vedia, H. Mach, L. M. Fraile, J. M. Udías, and S. Lalkovski, Nucl. Instrum. Methods A 795, 144 (2015).

[34] XIA LLC (2009), https://www.xia.com/.

[35] H. Mach, F. Wohn, G. Molnár, K. Sistemich, J. C. Hill, M. Moszyński, R. Gill, W. Krips, and D. Brenner, Nucl. Phys. A 523, 197 (1991).

[36] H. Mach and B. Fogelberg, Phys. Scr. 1995, 270 (1995).

[37] C. M. Baglin, Nucl. Data Sheets 109, 2257 (2008).

[38] P. Hoff and B. Fogelberg, Nucl. Phys. A 368, 210 (1981).

[39] D. Verney, B. Tastet, K. Kolos, F. Le Blanc, F. Ibrahim, M. Cheikh Mhamed, E. Cottereau, P. V. Cuong, F. Didierjean, G. Duchêne, S. Essabaa, M. Ferraton, S. Franchoo, L. H. Khiem, C. Lau, J.-F. Le Du, I. Matea, B. Mouginot, M. Niikura, B. Roussière, I. Stefan, D. Testov, and J.-C. Thomas, Phys. Rev. C 87, 054307 (2013).

[40] M. Wang, G. Audi, F. G. Kondev, W. Huang, S. Naimi, and X. Xu, Chin. Phys. C 41, 030003 (2017).
[41] D. Mcmillan and B. Pate, Nucl. Phys. A 174, 593 (1971).

[42] J. Kratz, H. Franz, N. Kaffrel, and G. Herrmann, Nucl. Phys. A 250, 13 (1975).

[43] B. Singh, Nucl. Data Sheets 96, 1 (2002).

[44] B. Singh, J. Rodríguez, S. Wong, and J. Tuli, Nucl. Data Sheets 84, 487 (1998).

[45] T. Kibédi, T. Burrows, M. Trzhaskovskaya, P. Davidson, and C. Nestor, Nucl. Instrum. Methods A 589, 202 (2008).

[46] B. Singh and J. Chen, Nucl. Data Sheets 116, 1 (2014).

[47] M.-G. Porquet, A. Astier, D. Verney, T. Venkova, I. Deloncle, F. Azaiez, A. Buta, D. Curien, O. Dorvaux, G. Duchêne, B. J. P. Gall, F. Khalfallah, I. Piqueras, M. Rousseau, M. Meyer, N. Redon, O. Stézowski, and A. Bogachev, Phys. Rev. C 84, 054305 (2011).

[48] W. Zoller, W. Walters, and G. Gordon, Nucl. Phys. A 142, 177 (1970).

[49] J. Diriken, I. Stefanescu, D. Balabanski, N. Blasi, A. Blazhev, N. Bree, J. Cederkäll, T. E. Cocolios, T. Davinson, J. Eberth, A. Ekström, D. V. Fedorov, V. N. Fedosseev, L. M. Fraile, S. Franchoo, G. Georgiev, K. Gladnishki, M. Huyse, O. V. Ivanov, V. S. Ivanov, J. Iwanicki, J. Jolie, T. Konstantinopoulos, T. Kröll, R. Krücken, U. Köster, A. Lagoyannis, G. Lo Bianco, P. Maierbeck, B. A. Marsh, P. Napiorkowski, N. Patronis, D. Pauwels, P. Reiter, M. Seliverstov, G. Sletten, J. Van de Walle, P. Van Duppen, D. Voulot, W. B. Walters, N. Warr, F. Wenander, and K. Wrzosek, Phys. Rev. C 82, 064309 (2010).

[50] B. Ekström, B. Fogelberg, P. Hoff, E. Lund, and A. Sangariyavanish, Phys. Scr. 34, 614 (1986).

[51] J. A. Winger, J. C. Hill, F. K. Wohn, R. L. Gill, X. Ji, and B. H. Wildenthal, Phys. Rev. C 38, 285 (1988).

[52] M. Zendel, N. Trautmann, and G. Herrmann, J. Inorg. Nucl. Chem. 42, 1387 (1980).

[53] F. K. Wohn, J. K. Halbig, W. L. Talbert, and J. R. McConnell, Phys. Rev. C 7, 160 (1973).

[54] A. F. Lisetskiy, B. A. Brown, M. Horoi, and H. Grawe, Phys. Rev. C 70, 044314 (2004).

[55] E. Caurier, G. Martínez-Pinedo, F. Nowacki, A. Poves, and A. P. Zuker, Rev. Mod. Phys. 77, 427 (2005).

[56] M. Honma, T. Otsuka, T. Mizusaki, and M. Hjorth-Jensen, Phys. Rev. C 80, 064323 (2009).

[57] P. C. Srivastava, J. Phys. G: Nucl. Part. Phys. 39, 015102 (2012).

[58] P. Vingerhoets, K. T. Flanagan, M. Avgoulea, J. Billowes, M. L. Bissell, K. Blaum, B. A. Brown, B. Cheal, M. De Rydt, D. H. Forest, C. Geppert, M. Honma, M. Kowalska, J. Krämer, A. Krieger, E. Mané, R. Neugart, G. Neyens, W. Nörtershäuser, T. Otsuka, M. Schug, H. H. Stroke, G. Tungate, and D. T. Yordanov, Phys. Rev. C 82, 064311 (2010)

[59] T. Otsuka, T. Suzuki, R. Fujimoto, H. Grawe, and Y. Akaishi, Phys. Rev. Lett. 95, 232502 (2005).

[60] P. Endt, At. Data Nucl. Data Tables 23, 547 (1979).

[61] T. Johnson and W. Kulp, Nucl. Data Sheets 129, 1 (2015).

[62] N. A. Smirnova, A. De Maesschalck, A. Van Dyck, and K. Heyde, Phys. Rev. C 69, 044306 (2004).

[63] J. M. Daugas, T. Faul, H. Grawe, M. Pfützner, R. Grzywacz, M. Lewitowicz, N. L. Achouri, J. C. Angélique, D. Baiborodin, R. Bentida, R. Béraud, C. Borcea, C. R. Bingham, W. N. 
Catford, A. Emsallem, G. de France, K. L. Grzywacz, R. C. Lemmon, M. J. Lopez Jimenez, F. de Oliveira Santos, P. H. Regan, K. Rykaczewski, J. E. Sauvestre, M. Sawicka, M. Stanoiu, K. Sieja, and F. Nowacki, Phys. Rev. C 81, 034304 (2010).
[64] J. Hakala, S. Rahaman, V.-V. Elomaa, T. Eronen, U. Hager, A. Jokinen, A. Kankainen, I. D. Moore, H. Penttilä, S. RintaAntila, J. Rissanen, A. Saastamoinen, T. Sonoda, C. Weber, and J. Äystö, Phys. Rev. Lett. 101, 052502 (2008).

[65] M. Caprio, Comput. Phys. Commun. 171, 107 (2005). 